\title{
Optimization of Novel Aza-benzimidazolone mGluR2 PAM's with respect to LLE and PK Properties and Mitigation of CYP TDI
}

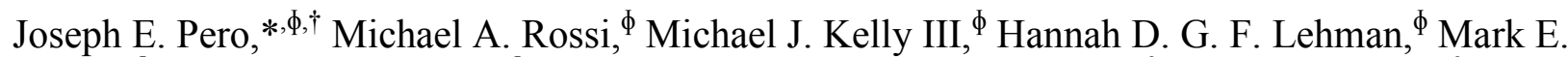
Layton, ${ }^{\phi}$ Robert M. Garbaccio, ${ }^{\phi}$ Julie A. O’Brien, ${ }^{\S}$ Brian C. Magliaro, ${ }^{\circ}$ Jason M. Uslaner, ${ }^{6}$ Sarah L. Huszar, ${ }^{\natural}$ Kerry L. Fillgrove, Cuyue Tang, ${ }^{\|}$Yuhsin Kuo," Leo A. Joyce, ${ }^{\Delta}$ Edward C. Sherer, ${ }^{\Psi}$ and Marlene A. Jacobson ${ }^{\text {f }}$

Departments of ${ }^{\phi}$ Medicinal Chemistry, ${ }^{\S}$ In Vitro Sciences, ${ }^{9}$ Psychiatry Research, ${ }^{6}$ Central Pharmacology, Drug Metabolism, ${ }^{\Delta}$ Process and Analytical Chemistry, and ${ }^{\Psi}$ Structural Chemistry, Merck Research Laboratories, P.O. Box 4, Sumneytown Pike, West Point, Pennsylvania 19486

\section{SUPPORTING INFORMATION}

Biological Assays: All animal studies were performed according to the NIH Guide for the Care and Use of Laboratory Animals, and experimental protocols were reviewed by the Merck Animal Care and Use Committee.

FLIPR Assay: CHOdhfr- cells co-expressing human mGluR2 receptors and the promiscuous Gprotein Ga16 were plated (40K cells per well) in clear-bottomed, poly-D-lysine-coated 384-well plates (BD Biosciences, Franklin Lakes, NJ) in glutamine/glutamine-free medium using a Multidrop384 cell dispenser (Thermo Electron Corporation, Waltham, MA). The plated cells were grown overnight at $37{ }^{\circ} \mathrm{C}$ in the presence of $6 \% \mathrm{CO}_{2}$. The next day, the cells were washed with $3 \times 100 \mu \mathrm{L}$ of assay buffer [Hanks' balanced salt solution (Invitrogen) containing $20 \mathrm{mM}$ HEPES (Invitrogen), $2.5 \mathrm{mM}$ probenecid (Sigma Chemical Co., St. Louis, MO) and 0.1\% bovine serum albumin (Sigma)] using an EMBLA cell washer (Molecular Devices Corp., Sunnyvale, CA). The cells were incubated with $2 \mu \mathrm{M}$ Fluo-4AM and 0.02\% Pluronic acid (Molecular Probes, Eugene, OR) for $1 \mathrm{~h}$ at $37{ }^{\circ} \mathrm{C}$ and $6 \% \mathrm{CO}_{2}$. The extracellular dye was removed by washing as described above. $\mathrm{Ca}^{2+}$ flux was measured using FLIPR384 fluorometric imaging 
plate reader (Molecular Devices Corp., Sunnyvale, CA). Compounds were serially diluted in $100 \%$ DMSO and then diluted in assay buffer to a 3x stock at $\%$ DMSO. This stock was then applied to cells for a final DMSO concentration of $0.67 \%$. For potency determination, the cells were preincubated with various concentrations of compound for $5 \mathrm{~min}$ and then stimulated for 3 min with an $\mathrm{EC}_{20}$ concentration of glutamate to measure potentiation. The peak of the calcium response was used to construct concentration response curves. For glutamate shift assays, concentrations of glutamate over a dose response range of $(0.01-10 \mu \mathrm{M})$ were employed.

Pharmacokinetic Studies: Male Wistar Hannover rats weighing approximately 200 to $300 \mathrm{~g}$ were obtained from Charles River (Raleigh, NC). Male beagle dogs weighing approximately 8 to $10 \mathrm{~kg}$ were obtained from Marshall Farms (North Rose, NY). All procedures were approved by the Merck Research Laboratories Institutional Animal Care and Use Committee. Rats and dogs were housed in temperature-controlled rooms with a 12-h light/dark cycle. Rats were surgically prepared with a cannula in the jugular vein for sample collection. For all studies the animals were deprived of food 14 to $18 \mathrm{~h}$ before dosing but were allowed free access to water. Food was provided to rats and dogs 6 to $8 \mathrm{~h}$ after dosing. Blood samples for the determination of test agent plasma concentration were collected in heparinized syringes at multiple time points up to $24 \mathrm{~h}$ after single dose administration and plasma was subsequently obtained by centrifugation. All samples were stored at $-20{ }^{\circ} \mathrm{C}$ until analyzed. Finally, all biological samples were centrifuged before analysis to remove particular matter.

Incubation with Human, Dog, and Rat Liver Microsomes: All incubations were performed in phosphate buffer (100 mM, pH 7.4) containing $\mathrm{MgCl}_{2}(10 \mathrm{mM})$. Each compound (10 $\left.\mu \mathrm{M}\right)$ was incubated with liver microsomes $(0.5 \mathrm{mg} / \mathrm{mL})$ for 30 to $60 \mathrm{~min}$ in the presence of both NADPH $(1 \mathrm{mM})$ and GSH $(5 \mathrm{mM})$. Reactions were terminated by adding one-half reaction volume of acetonitrile to precipitate proteins. After brief vortexing and centrifugation (10 $\mathrm{min}$ at $3800 \mathrm{x} \mathrm{g})$, the supernatant was subjected to vacuum centrifugation for 10 minutes to remove excess acetonitrile and the resulting solution was submitted for LC-HRMS analysis.

Sample Analysis: For metabolite identification, separation of metabolites and parent compound was achieved on a C18 Synergi MAX-RP 100A column $(2.0$ x 50 mm, $2.5 \mu \mathrm{m}$, Phenomenex, 
Torrance, CA) using a Water Acquity UPLC system (Waters, Milford, MA) with a flow rate of $0.5 \mathrm{~mL} / \mathrm{min}$. Solvent A consisted of $0.1 \%$ formic acid in water and solvent B consisted of acetonitrile containing $0.1 \%$ formic acid. The initial mobile phase of $5 \%$ solvent $\mathrm{B}$ was increased linearly to $15 \% \mathrm{~B}$ in $0.5 \mathrm{~min}$ followed by a linear increase to $50 \% \mathrm{~B}$ over 11.5 minutes. The gradient was then increased linearly to $90 \%$ solvent $\mathrm{B}$ and held at that value for an additional 1.5 minutes. The gradient was returned to $5 \%$ solvent $\mathrm{B}$ in 0.5 minutes and the column was re-equilibrated prior to the next injection. Mass spectrometric analysis was performed on a Waters Q-Tof Premier mass spectrometer equipped with an electrospray ionization source (Waters, Milford, MA) as described previously (Reference: Tiller, P.R.; Yu, S.; Castro-Perez, J.; Fillgrove, K.L.; Baillie, T.A. Rapid Commun. Mass Spectrom. 2008 Apr;22(7):1053-61).

Rat hyperlocomotion behavioral assay: Locomotor response to MK-801 (0.23 mpk, sc) over a 90 min session as a function of treatment with compounds 18 and $\mathbf{2 1}$ (10,30 mpk; po) or vehicle $(20 \% \mathrm{VitE} / \mathrm{TPGS})$ in male Sprague-Dawley rats $(250-300 \mathrm{~g})$ that had been pair-housed in standard laboratory conditions. Rats were placed in activity monitors and, after $30 \mathrm{~min}$ of habituation, treated with either vehicle or 18 and placed back into activity monitors. After an additional $30 \mathrm{~min}$, rodents were given an injection of saline or MK-801. Distance traveled over the following 90 min was summed.

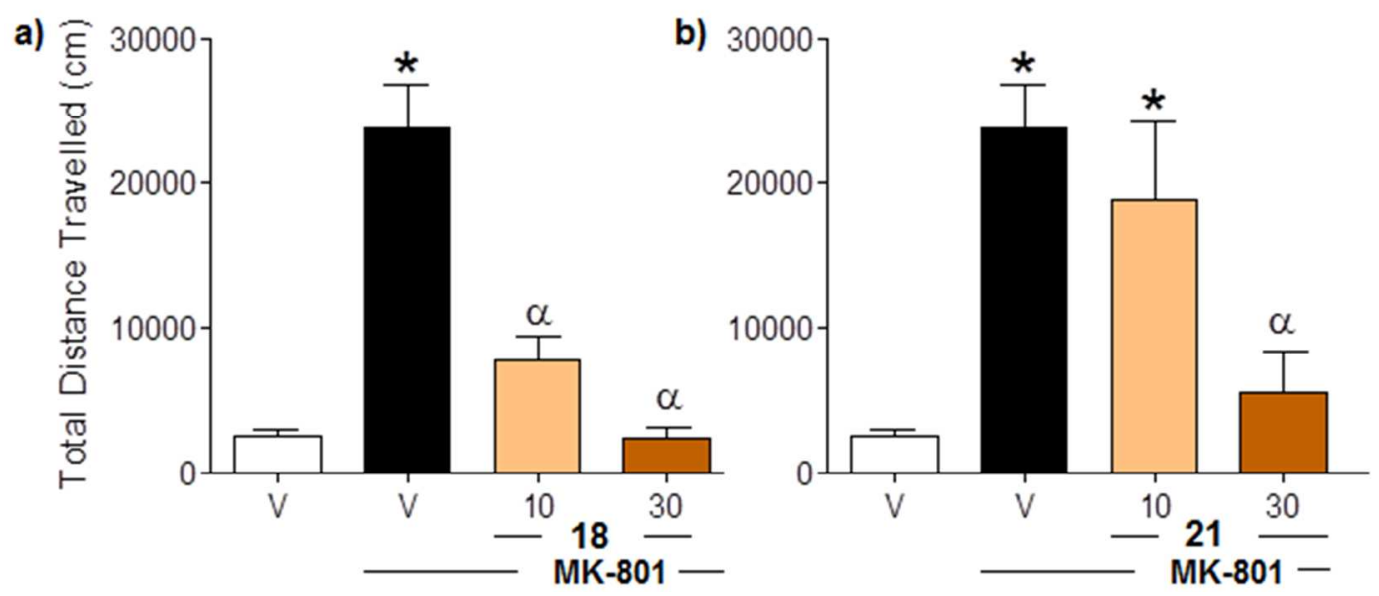




\section{Synthetic Methods:}

\section{Scheme 1: Synthesis of Intermediate 3}

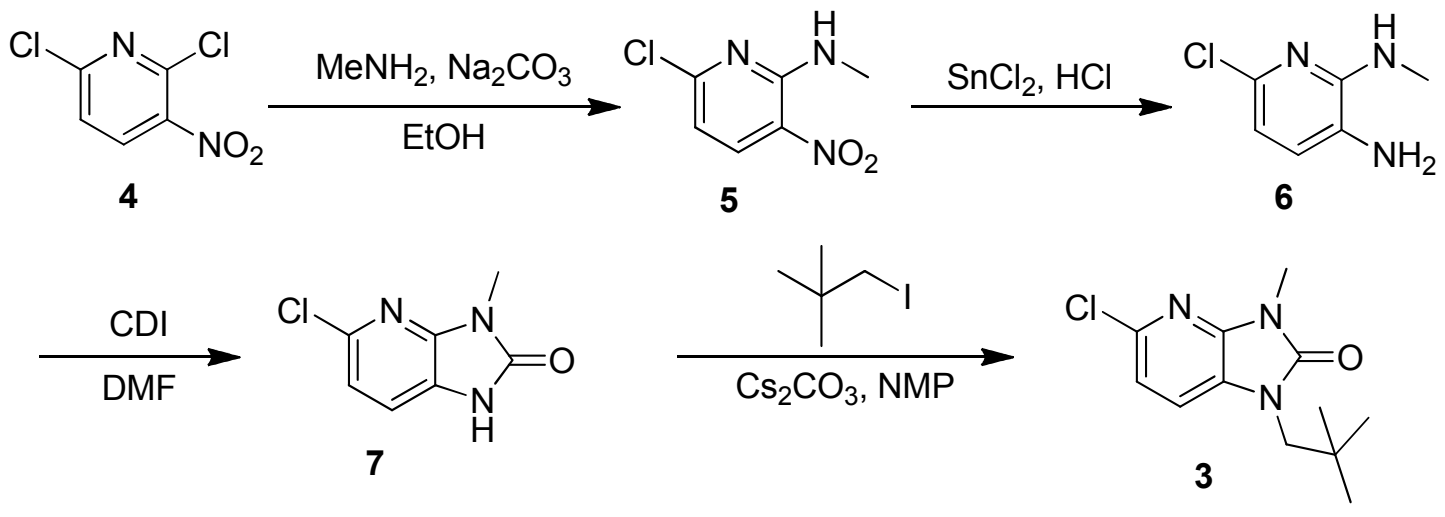

\section{6-Chloro- $N$-methyl-3-nitropyridin-2-amine (5):}

2,6-Dichloro-3-nitropyridine $(4,2.0 \mathrm{~g}, 10.4 \mathrm{mmol})$ and sodium carbonate $(2.75 \mathrm{~g}, 25.9 \mathrm{mmol})$ were suspended in ethanol $(100 \mathrm{~mL})$. Methylamine in methanol $(7.8 \mathrm{~mL}, 15.6 \mathrm{mmol}, 2 \mathrm{M})$ was then added and the resulting mixture stirred at room temperature for $3 \mathrm{~h}$. The yellow solution was concentrated in vacuo and then re-dissolved in ethyl acetate followed by washing with sodium bicarbonate and brine. The organic phase was dried over sodium sulfate, filtered and concentrated in vacuo. The yellow solid was then re-dissolved in ethanol and recrystalized to give 6-chloro- $N$-methyl-3-nitropyridin-2-amine (5) as a yellow solid (1.6 g, 82\%). LRMS m/z $(\mathrm{M}+\mathrm{H})$ : calculated $=187.6$; observed $=187.1 .{ }^{1} \mathrm{H}$ NMR $\left(400 \mathrm{MHz}, \mathrm{DMSO}-\mathrm{d}_{6}\right): \delta 3.00(3 \mathrm{H}, \mathrm{d}, \mathrm{J}$ $=5.1 \mathrm{~Hz}), 6.77(1 \mathrm{H}, \mathrm{d}, \mathrm{J}=8.1 \mathrm{~Hz}), 8.42(1 \mathrm{H}, \mathrm{d}, \mathrm{J}=8.1 \mathrm{~Hz}), 8.72(1 \mathrm{H}, \mathrm{m})$.

\section{6-Chloro- $N^{2}$-methylpyridine-2,3-diamine (6):}

6-Chloro- $N$-methyl-3-nitropyridin-2-amine $(5,10.5 \mathrm{~g}, 56 \mathrm{mmol})$ and tin(II) chloride dihydrate (50.5 g, $224 \mathrm{mmol})$ were suspended in concentrated $\mathrm{HCl}(80 \mathrm{~mL})$ and refluxed for $18 \mathrm{~h}$. The solution was cooled to $0{ }^{\circ} \mathrm{C}$ and $5 \mathrm{M}$ aqueous $\mathrm{NaOH}$ was cautiously added until the solution had a $\mathrm{pH}$ of 8 . The mixture was subsequently partitioned with $300 \mathrm{~mL}$ ethyl acetate and the layers were separated. The organics were washed with brine $(200 \mathrm{~mL})$, dried over sodium sulfate, 
filtered and concentrated in vacuo to give 6-chloro- $N^{2}$-methylpyridine-2,3-diamine (6) as an offwhite solid (7.5 g, 85\%). HRMS m/z $(\mathrm{M}+\mathrm{H})$ : calculated = 158.0480; observed = 158.0471.

\section{5-Chloro-3-methyl-1,3-dihydro-2H-imidazo[4,5-b]pyridin-2-one (7):}

6-Chloro- $N^{2}$-methylpyridine-2,3-diamine (6,35 g, $\left.222 \mathrm{mmol}\right)$ and 1,1'-carbonyldiimidazole (63 $\mathrm{g}, 389 \mathrm{mmol})$ were suspended in DMF $(150 \mathrm{~mL})$. The resulting reaction mixture was heated to $80{ }^{\circ} \mathrm{C}$ for $18 \mathrm{~h}$. The reaction contents were subsequently partitioned with ethyl acetate $(300 \mathrm{~mL})$ and saturated $\mathrm{NaHCO}_{3}(200 \mathrm{~mL})$. The layers were separated and the organic layer was washed with brine $(5 \times 100 \mathrm{~mL})$, dried over sodium sulfate, filtered and concentrated in vacuo to give 5chloro-3-methyl-1,3-dihydro-2 $\mathrm{H}$-imidazo[4,5-b]pyridin-2-one (7) as a white solid (39 g, 95\%). $\mathrm{HRMS} \mathrm{m} / \mathrm{z}(\mathrm{M}+\mathrm{H})$ : calculated $=184.0272 ;$ observed $=184.0279$.

\section{5-Chloro-1-(2,2-dimethylpropyl)-3-methyl-1,3-dihydro-2H-imidazo[4,5-b]pyridin-2-one (3):}

5-Chloro-3-methyl-1,3-dihydro-2H-imidazo[4,5-b]pyridin-2-one (7, $2.97 \mathrm{~g}, 16.2 \mathrm{mmol})$ and cesium carbonate (15.8 g, $48.6 \mathrm{mmol})$ were suspended in NMP $(25 \mathrm{~mL})$. Neopentyl iodide (6.4 g, $32.4 \mathrm{mmol}$ ) was added to the suspension and the resulting mixture was warmed to $90{ }^{\circ} \mathrm{C}$ and stirred for $15 \mathrm{~h}$. The reaction was then cooled to room temperature and partitioned with ethyl acetate $(200 \mathrm{~mL})$ and saturated $\mathrm{NaHCO}_{3}(150 \mathrm{~mL})$. The layers were separated and the organic layer was washed with brine $(5 \times 100 \mathrm{~mL})$, dried over sodium sulfate, filtered and concentrated in vacuo. Purification with normal-phase CombiFlash ISCO (80 g column, 0-60\% EtOAc:Hexanes) gave 5-chloro-1-(cyclopropylmethyl)-3-methyl-1,3-dihydro-2H-imidazo[4,5b]pyridin-2-one (2) as a tan solid (3.2 g, 78\%). HRMS m/z $(\mathrm{M}+\mathrm{H})$ : calculated = 253.7340; observed $=253.7341$. 


\section{Scheme 2: General Procedure for Palladium-catalyzed $\boldsymbol{N}$-arylations}
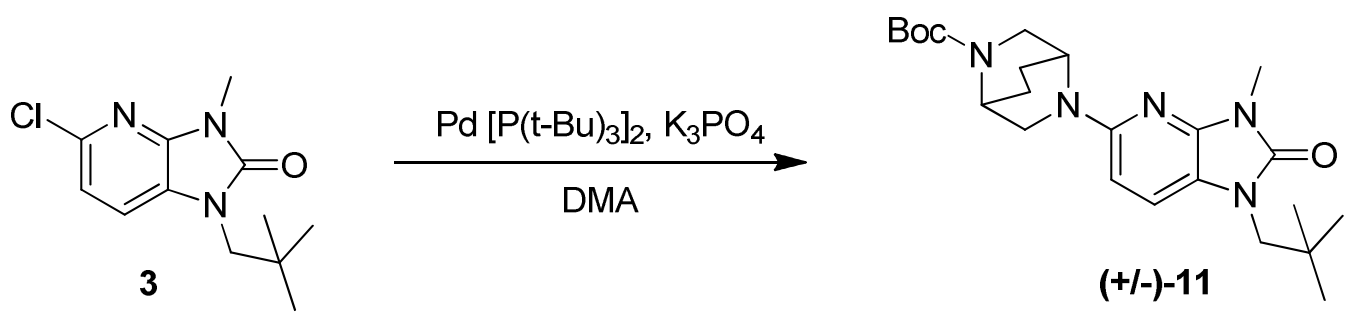

\section{(1+/-)-tert-Butyl 5-(3-methyl-1-neopentyl-2-oxo-2,3-dihydro-1H-imidazo[4,5-b]pyridin-5-}

yl)-2,5-diazabicyclo-[2.2.2]-octane-2-carboxylate $((+/-)-11)$ :

Anhydrous dimethylacetamide ( $85 \mathrm{~mL}$, degassed prior to use) was added to a mixture of 5chloro-1-(2,2-dimethylpropyl)-3-methyl-1,3-dihydro-2H-imidazo[4,5-b]pyridin-2-one (3, $17.9 \mathrm{~g}$, $70.5 \mathrm{mmol})$, potassium phosphate tribasic $(30.1 \mathrm{~g}, 142 \mathrm{mmol})$, bis(tri-tbutylphosphine)palladium(0) (1.45 g, $2.84 \quad \mathrm{mmol}), \quad$ and $\quad(1 S, \quad 4 S)$-tert-butyl-2,5diazabicyclo[2.2.2] octane-2-carboxylate $(15.81 \mathrm{~g}, 74.5 \mathrm{mmol})$. The resulting suspension was heated to $100{ }^{\circ} \mathrm{C}$ for $18 \mathrm{~h}$. Following this duration, the reaction contents were cooled to room temperature, filtered through Celite, washed with EtOAc and concentrated in vacuo. Purification with normal-phase CombiFlash ISCO (330 g column, 0-40\% EtOAc:Hexanes) afforded (1S,4S)tert-butyl-5-[1-(2,2-dimethylpropyl)-3-methyl-2-oxo-2,3-dihydro-1 $H$-imidazo[4,5-b]pyridin-5yl]-2,5-diazabicyclo[2.2.2] octane-2-carboxylate $((+/-)-11)$ as a white solid (26.3 g, 87\%). HRMS m/z $(\mathrm{M}+\mathrm{H})$ : calculated $=430.2813$; observed $=430.2807$. 
The following compounds were prepared from intermediate 3 using these conditions and the appropriate secondary amine:

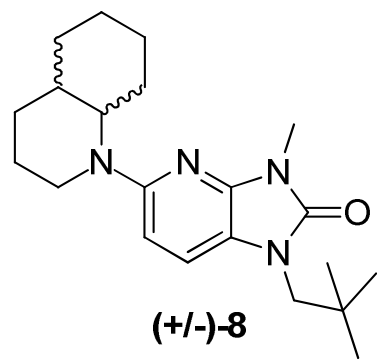

(+/-)3-Methyl-1-neopentyl-5-(octahydroquinolin-1(2H)-yl)-1H-imidazo[4,5-b]pyridin2(3H)-one ((+/-)-8) (mixture of cis/trans isomers):

$38 \%$ yield (white solid); HRMS m/z $(\mathrm{M}+\mathrm{H})$ : calculated $=357.2649$; observed $=357.2652 .{ }^{1} \mathrm{H}$ NMR (400 MHz, $\left.\mathrm{CDCl}_{3}\right): \delta$ 6.96-7.02 (m, $\left.2 \mathrm{H}\right) ; 6.21$ (d, J = 8.6 Hz, $\left.2 \mathrm{H}\right) ; 4.25(\mathrm{~d}, \mathrm{~J}=12.1 \mathrm{~Hz}$, $2 \mathrm{H}) ; 3.97(\mathrm{~d}, \mathrm{~J}=12.7 \mathrm{~Hz}, 2 \mathrm{H}) ; 3.62(\mathrm{~s}, 2 \mathrm{H}) ; 3.55(\mathrm{~s}, 4 \mathrm{H}) ; 3.40$ (s, $6 \mathrm{H}) ; 2.78-2.84$ (m, $2 \mathrm{H})$; 1.94-2.01 (m, $2 \mathrm{H}) ; 1.74-1.81(\mathrm{~m}, 6 \mathrm{H}) ; 1.60-1.64(\mathrm{~m}, 6 \mathrm{H}) ; 1.36-1.46$ (m, $6 \mathrm{H}) ; 1.00$ (m, 22 $\mathrm{H})$.

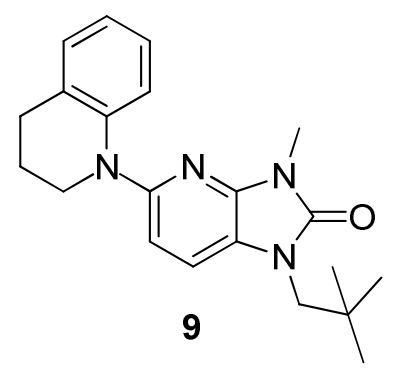

5-(3,4-Dihydroquinolin-1(2H)-yl)-3-methyl-1-neopentyl-1H-imidazo[4,5-b]pyridin-2(3H)one (9):

$79 \%$ yield (pale yellow solid); HRMS m/z $(\mathrm{M}+\mathrm{H})$ : calculated $=351.2179$; observed $=351.2180$. ${ }^{1} \mathrm{H}$ NMR (400 MHz, $\left.\mathrm{CDCl}_{3}\right): \delta 7.16(\mathrm{~d}, \mathrm{~J}=8.2 \mathrm{~Hz}, 1 \mathrm{H}) ; 6.99-7.11(\mathrm{~m}, 3 \mathrm{H}) ; 6.80-6.85$ (m, 2 $\mathrm{H}) ; 3.89(\mathrm{t}, \mathrm{J}=5.8 \mathrm{~Hz}, 2 \mathrm{H}) ; 3.61(\mathrm{~s}, 2 \mathrm{H}) ; 3.47(\mathrm{~s}, 3 \mathrm{H}) ; 2.83(\mathrm{t}, \mathrm{J}=6.5 \mathrm{~Hz}, 2 \mathrm{H}) ; 1.95-2.04$ $(\mathrm{m}, 2 \mathrm{H}) ; 1.04(\mathrm{~s}, 9 \mathrm{H})$. 


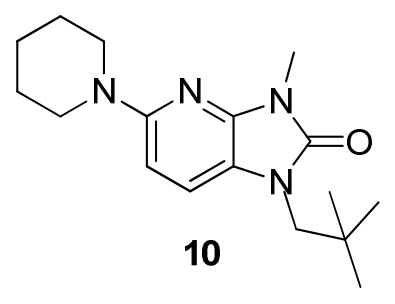

3-Methyl-1-neopentyl-5-(piperidin-1-yl)-1H-imidazo[4,5-b]pyridin-2(3H)-one (10):

97\% yield (white solid); HRMS m/z $(\mathrm{M}+\mathrm{H})$ : calculated $=303.2179$; observed $=303.2174 .{ }^{1} \mathrm{H}$ NMR (400 MHz, $\left.\mathrm{CDCl}_{3}\right): \delta$ 7.04-7.07 (m, $\left.1 \mathrm{H}\right) ; 6.28-6.31(\mathrm{~m}, 1 \mathrm{H}) ; 3.58(\mathrm{~s}, 2 \mathrm{H}) ; 3.42-3.46$ (m, $7 \mathrm{H}) ; 1.62-1.69(\mathrm{~m}, 6 \mathrm{H}) ; 1.01(\mathrm{~s}, 9 \mathrm{H})$.

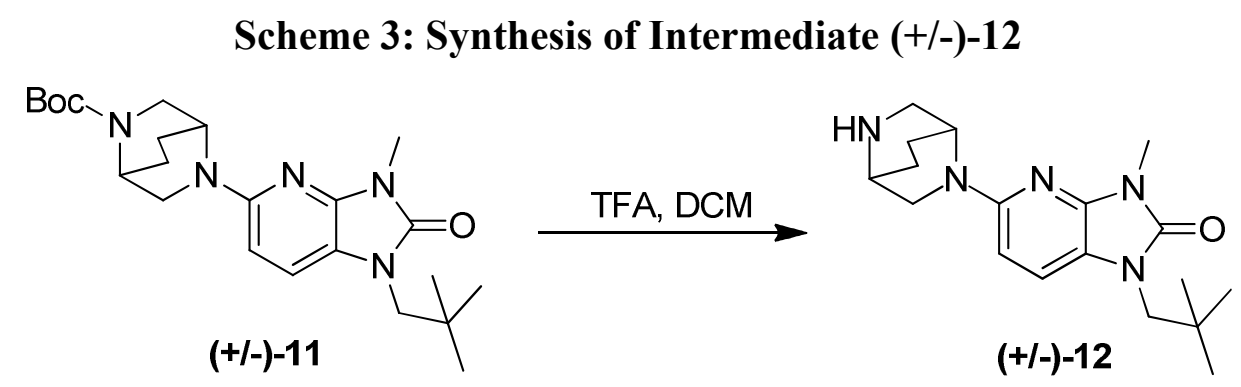

5-((+/-)-2,5-Diazabicyclo[2.2.2]octan-2-yl)-3-methyl-1-neopentyl-1H-imidazo[4,5-b]pyridin2(3H)-one $((+/-)-12):$

(+/-)-tert-Butyl-5-(3-methyl-1-neopentyl-2-oxo-2,3-dihydro-1H-imidazo[4,5-b]pyridin-5-yl)2,5-diazabicyclo-[2.2.2]-octane-2-carboxylate ((+/-)-11, $7.2 \mathrm{~g}, 16.8 \mathrm{mmol})$ was added to a mixture of dichloromethane $(137 \mathrm{~mL})$ and trifluoroacetic acid $(30.5 \mathrm{~mL})$ at room temperature. After stirring for $30 \mathrm{~min}$, the solvent was removed in vacuo. The resulting residue was then dissolved into ethyl acetate $(200 \mathrm{~mL})$ and washed sequentially with saturated sodium bicarbonate $(2 \times 100 \mathrm{~mL})$, water $(1 \times 100 \mathrm{~mL})$ and brine $(1 \times 100 \mathrm{~mL})$. The organics were dried over sodium sulfate, filtered and concentrated in vacuo to give 5-((+/-)-2,5-diazabicyclo[2.2.2]octan-2-yl)-3methyl-1-neopentyl-1H-imidazo[4,5-b]pyridin-2(3H)-one $((+/-)-12)$ as a white solid (5.4 g, 98\%). LRMS m/z $(\mathrm{M}+\mathrm{H})$ : calculated = 329.4; observed $=330.1$. 


\section{Scheme 4: Synthesis of Compound (+/-)-13}
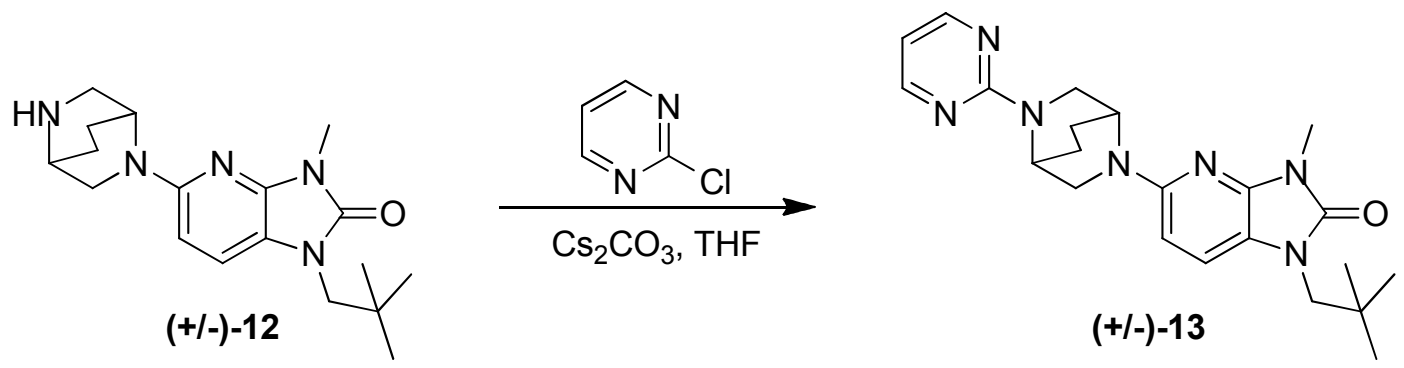

3-Methyl-1-neopentyl-5-((+/-)-5-(pyrimidin-2-yl)-2,5-diazabicyclo[2.2.2]octan-2-yl)-1Himidazo[4,5-b]pyridin-2(3H)-one $((+/-)-13)$ :

5-((1S,4S)-2,5-Diazabicyclo[2.2.2]octan-2-yl)-3-methyl-1-neopentyl-1H-imidazo[4,5-b]pyridin2(3H)-one $((+/-)-\mathbf{1 2}, 30 \mathrm{mg}, 0.091 \mathrm{mmol})$ and cesium carbonate $(89 \mathrm{mg}, 0.273 \mathrm{mmol})$ were added to anhydrous THF $(911 \mu \mathrm{L})$. To this reaction mixture was added 2-chloro-pyrimidine $(10.4 \mathrm{mg}, 0.091 \mathrm{mmol})$ and the contents were stirred for $18 \mathrm{~h}$ at room temperature. Following this duration, the solvent was removed in vacuo and the resulting residue was partitioned between ethyl acetate $(5 \mathrm{~mL})$ and water $(5 \mathrm{~mL})$. The layers were separated and the aqueous layer was extracted with ethyl acetate $(3 \times 3 \mathrm{~mL})$. The combined organics were dried over sodium sulfate, filtered and concentrated in vacuo to give an orange residue. Purification via reversephase HPLC (20 min gradient, $10-100 \% \mathrm{CH}_{3} \mathrm{CN}: \mathrm{H}_{2} \mathrm{O}, 0.1 \%$ TFA as modifier) to give (following EtOAc/saturated $\mathrm{NaHCO}_{3}$ workup of product-containing fractions) 3-methyl-1-neopentyl-5-((+/)-5-(pyrimidin-2-yl)-2,5-diazabicyclo[2.2.2]octan-2-yl)-1H-imidazo[4,5-b]pyridin-2(3H)-one $((+/-)-\mathbf{1 3})$ as a white solid $(13 \mathrm{mg}, 34 \%)$. HRMS $\mathrm{m} / \mathrm{z}(\mathrm{M}+\mathrm{H})$ : calculated $=408.25$; observed $=$ 408.25. ${ }^{1} \mathrm{H}$ NMR (400 MHz, $\left.\mathrm{CDCl}_{3}\right): \delta 8.31$ (s, $\left.2 \mathrm{H}\right) ; 7.07$ (d, J = 8.4 Hz, $\left.1 \mathrm{H}\right) ; 6.48(\mathrm{t}, \mathrm{J}=4.8$ $\mathrm{Hz}, 1 \mathrm{H}) ; 5.99(\mathrm{~d}, \mathrm{~J}=8.5 \mathrm{~Hz}, 1 \mathrm{H}) ; 5.01-5.13(\mathrm{~m}, 2 \mathrm{H}) ; 3.83-3.87(\mathrm{~m}, 1 \mathrm{H}) ; 3.67-3.76(\mathrm{~m}, 2 \mathrm{H})$; 3.52-3.58 (m, 3 H); 3.42 (s, 3 H); 2.07-2.19 (m, 2 H); 1.87-1.99 (m, 2 H); 1.01 (s, 9 H). 


\section{Scheme 5: Synthesis of Compound (+/-)-14}

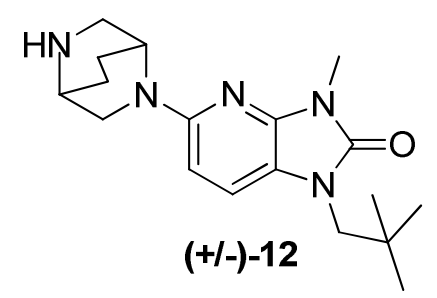

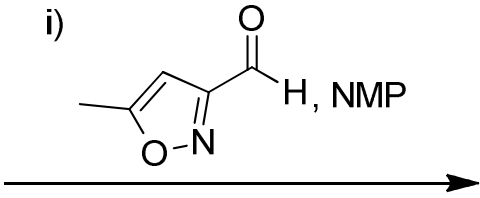

ii) $\mathrm{NaBH}(\mathrm{OAc})_{3}$

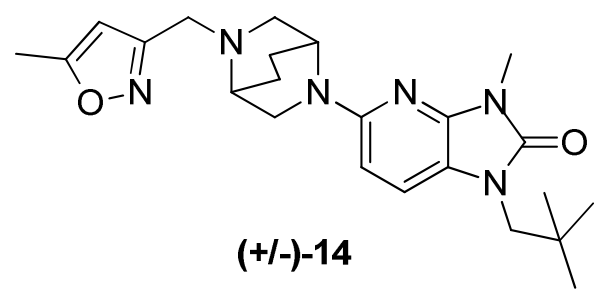

3-Methyl-5-((+/-)-5-((5-methylisoxazol-3-yl)methyl)-2,5-diazabicyclo[2.2.2]octan-2-yl)-1neopentyl-1H-imidazo $[4,5$-b]pyridin-2(3H)-one $((+/-)-14)$ :

5-((+/-)-2,5-Diazabicyclo[2.2.2] octan-2-yl)-3-methyl-1-neopentyl-1H-imidazo[4,5-b]pyridin2(3H)-one ((+/-)-12, $30 \mathrm{mg}, 0.091 \mathrm{mmol}), 5$-methylisoxazole-3-carbaldehyde (11.1 mg, 0.100 mmol), and acetic acid $(10.4 \mu \mathrm{L}, 0.182 \mathrm{mmol})$ were added to anhydrous NMP $(1 \mathrm{~mL})$ and the resulting solution was stirred at room temperature for $30 \mathrm{~min}$. Following this duration, sodium triacetoxyborohydride $(23.2 \mathrm{mg}, 0.109 \mathrm{mmol})$ was added. The resulting reaction mixture was stirred at room temperature for an additional $18 \mathrm{~h}$. The contents were then filtered and purified by reverse-phase HPLC (20 min gradient, $10-100 \% \mathrm{CH}_{3} \mathrm{CN}: \mathrm{H}_{2} \mathrm{O}, 0.1 \%$ TFA as modifier) to give (following EtOAc/saturated $\mathrm{NaHCO}_{3}$ workup of product-containing fractions) 3-methyl-5-((+/-)5-((5-methylisoxazol-3-yl)methyl)-2,5-diazabicyclo[2.2.2]octan-2-yl)-1-neopentyl-1 $H$ imidazo[4,5-b]pyridin-2(3H)-one ((+/-)-14) as a white solid (16 mg, 41\%). HRMS m/z (M+H): calculated $=425.2660$; observed $=425.2652 .{ }^{1} \mathrm{H}$ NMR $\left(400 \mathrm{MHz}, \mathrm{CDCl}_{3}\right): \delta 7.04(\mathrm{~d}, \mathrm{~J}=8.5 \mathrm{~Hz}$, 1 H); 5.93-6.03 (m, 2 H); 4.64 (s, 1 H); 3.68-3.75 (m, 3 H); 3.54 (s, 2 H); 3.36 (s, 3 H); 3.25 (d, J $=10.2 \mathrm{~Hz}, 1 \mathrm{H}) ; 3.03(\mathrm{~d}, \mathrm{~J}=10.2 \mathrm{~Hz}, 1 \mathrm{H}) ; 2.87-2.99(\mathrm{~m}, 2 \mathrm{H}) ; 2.37$ (s, $3 \mathrm{H}) ; 2.02-2.14(\mathrm{~m}, 1$ H); 1.77-1.98 (m, 2 H); 1.58-1.71 (m, 1 H); 0.98 (s, 9 H). 


\section{Scheme 6: Synthesis of Compound (+/-)-15}
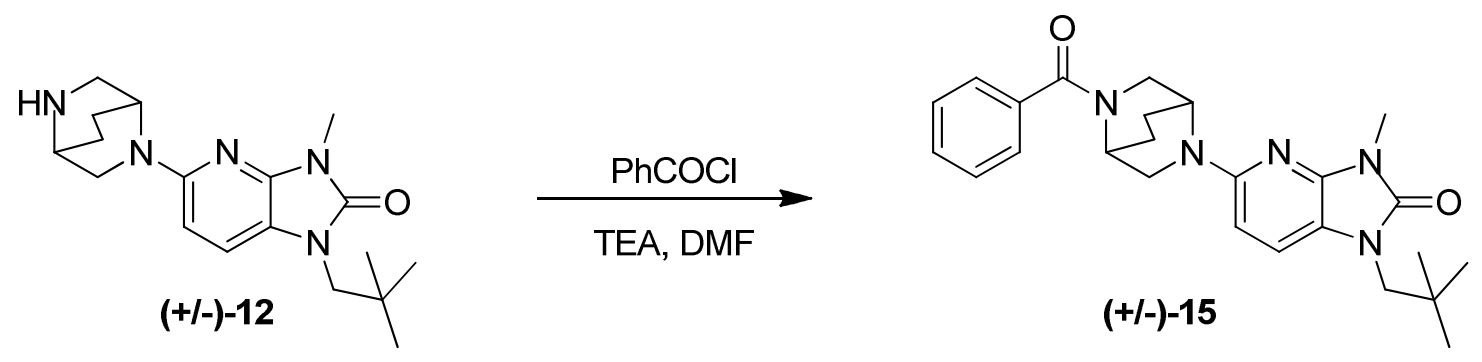

5-((+/-)-5-Benzoyl-2,5-diazabicyclo[2.2.2]octan-2-yl)-3-methyl-1-neopentyl-1H-imidazo[4,5b]pyridin-2(3H)-one $((+/-)-15)$ :

5-((+/-)-2,5-Diazabicyclo[2.2.2]octan-2-yl)-3-methyl-1-neopentyl-1H-imidazo[4,5-b]pyridin2(3H)-one ((+/-)-12, $1.5 \mathrm{~g}, 4.6 \mathrm{mmol})$ was added to anhydrous dimethylformamide $(45.5 \mathrm{~mL})$. Triethylamine $(1.27 \mathrm{~mL}, 9.11 \mathrm{mmol})$ and benzoyl chloride $(0.58 \mathrm{~mL}, 5.01 \mathrm{mmol})$ were then added, and the resulting solution was stirred at room temperature for $3 \mathrm{~h}$. Following this duration, the contents were filtered and the resulting filtrate was purified via reverse-phase HPLC (20 min gradient, 10-100\% $\mathrm{CH}_{3} \mathrm{CN}: \mathrm{H}_{2} \mathrm{O}, 0.1 \%$ TFA as modifier) to give (following EtOAc/saturated $\mathrm{NaHCO}_{3}$ workup of product-containing fractions) 5-((+/-)-5-benzoyl-2,5diazabicyclo[2.2.2] octan-2-yl)-3-methyl-1-neopentyl-1H-imidazo[4,5-b]pyridin-2(3H)-one $((+/-$ )-15) as a white solid (1.2 g, 61\%). HRMS m/z $(\mathrm{M}+\mathrm{H})$ : calculated $=434.2551$; observed = 434.2550. ${ }^{1} \mathrm{H} \mathrm{NMR}\left(400 \mathrm{MHz}, \mathrm{CDCl}_{3}\right): \delta$ 7.37-7.43 (m, $\left.5 \mathrm{H}\right) ;$ 7.03-7.07 (m, $\left.1 \mathrm{H}\right) ;$ 5.92-5.97 (m, $1 \mathrm{H}) ; 4.86-5.01$ (br s, $1 \mathrm{H}) ; 4.02(\mathrm{~s}, 1 \mathrm{H}) ; 3.84-3.92$ (m, $1 \mathrm{H}) ; 3.59-3.79$ (m, $2 \mathrm{H}) ; 3.47-3.55$ (m, $3 \mathrm{H}) ; 3.29-3.40$ (m, $3 \mathrm{H}), 1.70-2.23$ (m, $4 \mathrm{H})$; 0.84 (s, $9 \mathrm{H})$.

\section{Scheme 7: Synthesis of Compound $(1 S, 4 S)-18$}
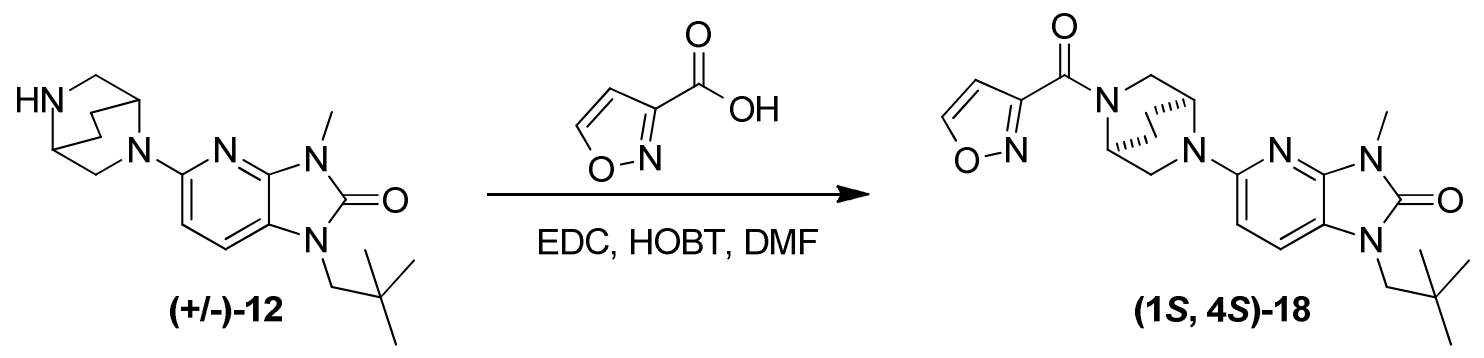

5-((1S,4S)-5-(Isoxazole-3-carbonyl)-2,5-diazabicyclo[2.2.2]octan-2-yl)-3-methyl-1neopentyl-1H-imidazo $[4,5$-b]pyridin-2(3H)-one $((1 S, 4 S)-18)$ :

5-((+/-)-2,5-Diazabicyclo[2.2.2]octan-2-yl)-3-methyl-1-neopentyl-1H-imidazo[4,5-b]pyridin2(3H)-one $((+/-)-12,35 \mathrm{mg}, 0.106 \mathrm{mmol})$ was added to anhydrous dimethylformamide $(1 \mathrm{~mL})$. 
Isoxazole-3-carboxylic acid (12.0 mg, $0.106 \mathrm{mmol})$, EDC (20.37 mg, $0.106 \mathrm{mmol})$, HOBT $(16.27 \mathrm{mg}, 0.106 \mathrm{mmol})$ and triethylamine $(30 \mu \mathrm{L}, 0.212 \mathrm{mmol})$ were added sequentially and the resulting reaction mixture was allowed to stir at room temperature for $18 \mathrm{~h}$. Following this duration, the contents were filtered and the resulting filtrate was purified via reverse-phase HPLC (20 min gradient, $10-100 \% \mathrm{CH}_{3} \mathrm{CN}: \mathrm{H}_{2} \mathrm{O}, 0.1 \%$ TFA as modifier) to give (following EtOAc:saturated $\mathrm{NaHCO}_{3}$ workup of product-containing fractions) 5-((+/-)-5-(isoxazole-3carbonyl)-2,5-diazabicyclo[2.2.2]octan-2-yl)-3-methyl-1-neopentyl-1 $H$-imidazo[4,5-b]pyridin2(3H)-one ((+/-)-18) as a white solid (37 mg, 82\%). Chiral HPLC (Chiralcel OD-H column, $\mathrm{MeOH}$ as eluent) provided 5-((1S,4S)-5-(isoxazole-3-carbonyl)-2,5-diazabicyclo[2.2.2]octan-2yl)-3-methyl-1-neopentyl-1H-imidazo[4,5-b]pyridin-2(3H)-one $((1 S, 4 S)$-18) as a white solid (15 $\mathrm{mg})$. HRMS $\mathrm{m} / \mathrm{z}(\mathrm{M}+\mathrm{H})$ : calculated $=425.2296$; observed $=425.2301 .{ }^{1} \mathrm{H}$ NMR $(400 \mathrm{MHz}$, $\left.\mathrm{CDCl}_{3}\right): \delta$ 8.41-8.45 (m, $\left.1 \mathrm{H}\right) ; 7.05(\mathrm{~d}, \mathrm{~J}=8.4 \mathrm{~Hz}, 1 \mathrm{H}) ; 6.73-6.75(\mathrm{~m}, 1 \mathrm{H}) ; 5.96(\mathrm{~d}, \mathrm{~J}=8.4$ Hz, 1 H); 4.98-5.06 (m, 2 H); 4.02-4.13 (m, 1 H); 3.66-3.89 (m, 2 H); 3.46-3.57 (m, 3 H); 3.38 (d, J = 8.3 Hz, $3 \mathrm{H}) ; 2.07-2.18$ (m, $2 \mathrm{H}) ; 1.85-1.96$ (m, $2 \mathrm{H}) ; 0.82$ (s, $9 \mathrm{H})$.

The following compounds were prepared from intermediate (+/-)-12 using these conditions and the appropriate carboxylic acid:

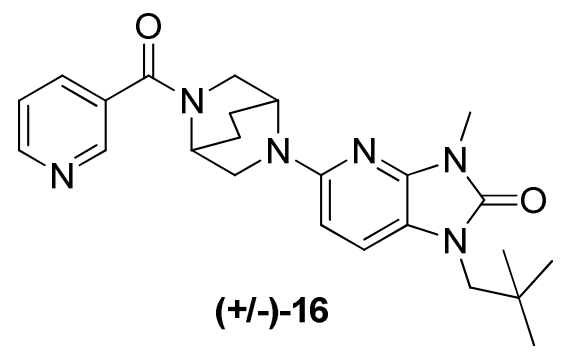

3-Methyl-1-neopentyl-5-((+/-)-5-nicotinoyl-2,5-diazabicyclo[2.2.2]octan-2-yl)-1Himidazo $[4,5-b]$ pyridin-2(3H)-one $((+/-)-16)$ :

$52 \%$ yield (white solid); HRMS $\mathrm{m} / \mathrm{z}(\mathrm{M}+\mathrm{H})$ : calculated $=435.2503$; observed $=435.2504 .{ }^{1} \mathrm{H}$ NMR (400 MHz, $\left.\mathrm{CDCl}_{3}\right): \delta$ 8.61-8.72 (m, $\left.2 \mathrm{H}\right) ; 7.74-7.81$ (m, $\left.1 \mathrm{H}\right) ; 7.29-7.69(\mathrm{~m}, 1 \mathrm{H}) ; 7.04-$ 7.07 (m, 1 H); 5.93-5.98 (m, 1 H); 4.90-5.03 (br s, 1 H); 4.00 (s, 1 H); 3.87-3.94 (m, 1 H); 3.643.80 (m, 2 H); 3.52-3.55 (m, 3 H); 3.39-3.44 (m, 2 H); 3.33 (s, 1 H); 1.78-2.16 (m, 4 H); 0.99 (s, $9 \mathrm{H})$. Note: Chiral HPLC omitted for this compound. It is a racemate. 


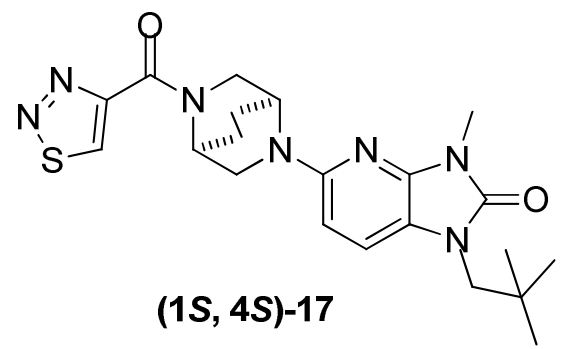

5-((1S,4S)-5-(1,2,3-Thiadiazole-4-carbonyl)-2,5-diazabicyclo[2.2.2]octan-2-yl)-3-methyl-1neopentyl-1 $H$-imidazo $[4,5$-b]pyridin-2 $(3 H)$-one $((1 S, 4 S)-17)$ :

56\% yield (white solid, racemate); Chiral HPLC (Chiralcel OD-H column, $\mathrm{MeOH}$ as eluent) provided $(1 S, 4 S)$-eutomer ( white solid). $\mathrm{HRMS} \mathrm{m} / \mathrm{z}(\mathrm{M}+\mathrm{H})$ : calculated $=442.202$; observed $=$ 442.2018. ${ }^{1} \mathrm{H}$ NMR (ppm) $\left(400 \mathrm{MHz}, \mathrm{CDCl}_{3}\right): \delta 9.20(\mathrm{~d}, \mathrm{~J}=7.2 \mathrm{~Hz}, 1 \mathrm{H}) ; 7.06(\mathrm{~d}, \mathrm{~J}=8.4 \mathrm{~Hz}$, $1 \mathrm{H}) ; 6.03$ (dd, J = 8.4, $2.4 \mathrm{~Hz}, 1 \mathrm{H}) ; 4.98-5.09$ (m, $1 \mathrm{H})$; 4.21-4.33 (m, $1 \mathrm{H}) ; 3.93-4.00$ (m, 1 H); 3.74-3.88 (m, 2 H); 3.50-3.61 (m, 3 H); 3.38 (d, J = 11.4 Hz, 3 H); 2.10-2.29 (m, 2 H); 1.881.99 (m, $2 \mathrm{H}) ; 0.98$ (d, J = 2.8 Hz, $9 \mathrm{H})$.

\section{Scheme 8: Synthesis of Compound $(1 S, 4 R)-21$}
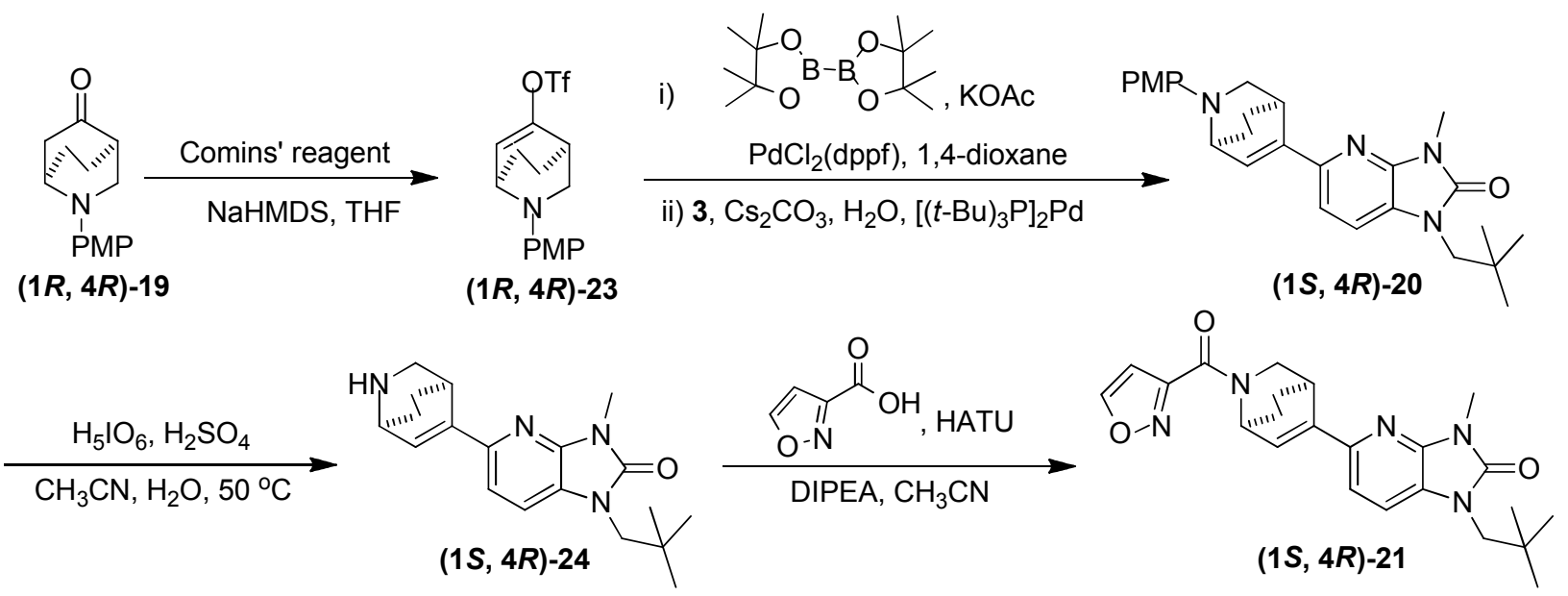

$(1 R, 4 R)-2$-(4-Methoxyphenyl)-2-azabicyclo[2.2.2]oct-5-en-5-yl trifluoromethanesulfonate $\underline{(1 R, 4 R)-23):}$

Known (Cordova, A. et al., Angew. Chem. Int. Ed. 2005, 117, 4955) (1R,4R)-2-(4Methoxyphenyl)-2-azabicyclo[2.2.2] octan-5-one-2-(4-Methoxyphenyl)-2-azabicyclo[2.2.2]octan-5-one $((1 R, 4 R)-19,4.0 \mathrm{~g}, 17.3 \mathrm{mmol})$ was added to anhydrous THF $(87 \mathrm{~mL})$ and cooled to $0{ }^{\circ} \mathrm{C}$. To this solution was added NaHMDS (10.4 mL, $20.8 \mathrm{mmol}, 2 \mathrm{M}$ solution in THF) followed by $\mathrm{N}$-(5-chloro-2-pyridyl)triflimide (Comins' reagent, $9.5 \mathrm{~g}, 24.2 \mathrm{mmol}$ ) in two equal 
portions. After $60 \mathrm{~min}$ at $0{ }^{\circ} \mathrm{C}$, LCMS showed consumption of starting material. The reaction was quenched with brine $(80 \mathrm{~mL})$ and diluted with ethyl acetate $(150 \mathrm{~mL})$ and water $(150 \mathrm{~mL})$. The layers were separated and the aqueous layer was back-extracted with ethyl acetate ( $3 \times 100$ $\mathrm{mL}$ ). The combined organics were dried over $\mathrm{Na}_{2} \mathrm{SO}_{4}$, filtered and concentrated in vacuo to give a red oil. Purification by normal-phase CombiFlash ISCO (120 g column, 0-40\% EtOAc:Hexanes) afforded $\quad(1 R, 4 R)$-2-(4-methoxyphenyl)-2-azabicyclo[2.2.2]oct-5-en-5-yl trifluoromethan-esulfonate $((1 R, 4 R)-\mathbf{2 3})$ as a pale yellow solid $(6.2 \mathrm{~g}, 98 \%)$, which was used immediately.

5-((1S,4R)-2-(4-Methoxyphenyl)-2-azabicyclo[2.2.2]oct-5-en-5-yl)-3-methyl-1-neopentyl$1 H$-imidazo[4,5-b]pyridin-2(3H)-one $((1 S, 4 R)-20)$ :

$(1 R, 4 R)$-2-(4-Methoxyphenyl)-2-azabicyclo[2.2.2] oct-5-en-5-yl trifluoromethanesulfonate ((1R, 4R)-23, $6.2 \mathrm{~g}, 17.1 \mathrm{mmol})$, bis(pinacolato)diboron (4.8 g, $18.8 \mathrm{mmol})$, potassium acetate $(5.0 \mathrm{~g}$, $51.2 \mathrm{mmol}$, ) and $\mathrm{PdCl}_{2}(\mathrm{dppf})(0.87 \mathrm{~g}, 1.2 \mathrm{mmol})$ were added to anhydrous 1,4-dioxane $(22 \mathrm{~mL})$ and heated to $60{ }^{\circ} \mathrm{C}$. After $18 \mathrm{~h}$, the reaction contents were cooled to RT, followed by the subsequent addition of water $(4.3 \mathrm{~mL}), 5$-chloro-1-(2,2-dimethylpropyl)-3-methyl-1,3-dihydro$2 \mathrm{H}$-imidazo[4,5-b]pyridin-2-one $(3,4.3 \mathrm{~g}, 17.1 \mathrm{mmol}), \mathrm{Cs}_{2} \mathrm{CO}_{3}(11.2 \mathrm{~g}, 34.3 \mathrm{mmol})$ and bis(tritert-butylphosphine)palladium(0) $(1.1 \mathrm{~g}, 2.22 \mathrm{mmol})$. The resulting mixture was heated to $60{ }^{\circ} \mathrm{C}$ for $4.5 \mathrm{~h}$. Following this duration, LCMS showed consumption of starting material. The contents were then cooled to room temperature, diluted with ethyl acetate $(100 \mathrm{~mL})$, filtered through Celite and rinsed with ethyl acetate $(3 \times 70 \mathrm{~mL})$ and water $(1 \times 30 \mathrm{~mL})$. The filtrate layers were separated and the combined organics were washed with saturated $\mathrm{NaHCO}_{3}(120$ $\mathrm{mL})$. The combined aqueous layers were then back-extracted with ethyl acetate $(2 \times 50 \mathrm{~mL})$. The combined organics were dried over $\mathrm{Na}_{2} \mathrm{SO}_{4}$, filtered and concentrated in vacuo to give a dark red oil. Purification by normal-phase CombiFlash ISCO (120 g column, 0-50\% EtOAc:Hex) afforded 5-((1S,4R)-2-(4-methoxyphenyl)-2-azabicyclo[2.2.2]oct-5-en-5-yl)-3methyl-1-neopentyl-1H-imidazo[4,5-b]pyridin-2(3H)-one $((1 S, 4 R)-20)$ as an off-white solid (4.4 g, 59\%). HRMS m/z (M+H): calculated = 433.2598; observed = 433.2591. 
5-((1S,4R)-2-Azabicyclo[2.2.2] oct-5-en-5-yl)-3-methyl-1-neopentyl-1H-imidazo $[4,5-$ blpyridin-2(3H)-one $((1 S, 4 R)-24)$ :

5-((1S,4R)-2-(4-Methoxyphenyl)-2-azabicyclo[2.2.2]oct-5-en-5-yl)-3-methyl-1-neopentyl-1Himidazo[4,5-b]pyridin-2(3H)-one $((1 S, 4 R)-20,339 \mathrm{mg}, 0.78 \mathrm{mmol})$ was added to $\mathrm{CH}_{3} \mathrm{CN}(7$ $\mathrm{mL})$ and water $(7 \mathrm{~mL})$. To this suspension was added aqueous $\mathrm{H}_{2} \mathrm{SO}_{4}(0.78 \mathrm{~mL}, 0.78 \mathrm{mmol}, 1.0$ M) followed by periodic acid in one portion $(179 \mathrm{mg}, 0.78 \mathrm{mmol})$. The reaction contents were heated to $50{ }^{\circ} \mathrm{C}$ for $18 \mathrm{~h}$. Following this duration, LCMS showed consumption of starting material. The mixture was subsequently filtered through Celite, rinsed with $\mathrm{CH}_{3} \mathrm{CN}$ and purified by reverse-phase HPLC (20 min gradient, 10-100\% $\mathrm{CH}_{3} \mathrm{CN}: \mathrm{H}_{2} \mathrm{O}, 0.1 \%$ TFA as modifier) to give (following EtOAc/saturated $\mathrm{NaHCO}_{3}$ workup of product-containing fractions) 5-((1S,4R)-2azabicyclo[2.2.2] oct-5-en-5-yl)-3-methyl-1-neopentyl-1 $H$-imidazo[4,5-b]pyridin-2(3H)-one $((1 S, 4 R)-24)$ as an off-white solid (74 mg, 29\%). HRMS m/z $(\mathrm{M}+\mathrm{H})$ : calculated = 327.2179; observed $=327.2187$.

5-((1S,4R)-2-(Isoxazole-3-carbonyl)-2-azabicyclo[2.2.2] oct-5-en-5-yl)-3-methyl-1-neopentyl$1 H$-imidazo[4,5-b]pyridin-2(3H)-one $((1 S, 4 R)-21)$ :

5-((1S,4R)-2-Azabicyclo[2.2.2] oct-5-en-5-yl)-3-methyl-1-neopentyl-1H-imidazo[4,5-b]pyridin2(3H)-one ((1S, 4R)-24, $291 \mathrm{mg}, 0.90 \mathrm{mmol})$, isoxazole-3-carboxylic acid (151 $\mathrm{mg}, 1.4 \mathrm{mmol})$, DIPEA (0.47 mL, $2.7 \mathrm{mmol})$ and HATU (510 mg, $1.4 \mathrm{mmol})$ were added to anhydrous 1,4dioxane $(9 \mathrm{~mL})$. After stirring for $10 \mathrm{~min}$ at room temperature, LCMS showed consumption of starting material. Purification by reverse-phase HPLC (20 min gradient, $20-100 \% \mathrm{CH}_{3} \mathrm{CN}: \mathrm{H}_{2} \mathrm{O}$, $0.1 \%$ TFA as modifier) to give (following EtOAc/saturated $\mathrm{NaHCO}_{3}$ workup of productcontaining fractions) 5-((1S,4R)-2-(isoxazole-3-carbonyl)-2-azabicyclo[2.2.2]oct-5-en-5-yl)-3methyl-1-neopentyl-1H-imidazo-[4,5-b]pyridin-2(3H)-one $((1 S, 4 R)-21)$ as a white solid (303 $\mathrm{mg}, 80 \%)$. HRMS m/z $(\mathrm{M}+\mathrm{H})$ : calculated $=422.2187$; observed $=422.2189 .{ }^{1} \mathrm{H} \mathrm{NMR}(400$ $\left.\mathrm{MHz}, \mathrm{CDCl}_{3}\right): \delta 8.40(1 \mathrm{H}, \mathrm{dd}, \mathrm{J}=27.77,1.66 \mathrm{~Hz}), 7.22-7.17(1 \mathrm{H}, \mathrm{m}), 7.12(1 \mathrm{H}, \mathrm{dd}, \mathrm{J}=8.11$, $1.50 \mathrm{~Hz}), 6.97(1 \mathrm{H}, \mathrm{m}), 6.70(1 \mathrm{H}, \mathrm{dd}, \mathrm{J}=11.92,1.66 \mathrm{~Hz}), 5.55-5.52(0.4 \mathrm{H}, \mathrm{m}), \quad 5.42-5.38(0.6$ H, m), $3.95(0.4$ H, m), 3.88 (1 H, m), $3.62(2.6$ H, m), $3.53(0.4$ H, m), 3.47 (3 H, d, J = 3.86 Hz), 3.36 (0.6 H, d, J = 12.39 Hz), 2.21-2.11 (1 H, m), 1.91-1.80 (1 H, m), 1.61-1.52 (2 H, m) $0.99(9 \mathrm{H}, \mathrm{d}, \mathrm{J}=1.91 \mathrm{~Hz})$. (Mixture of rotamers at RT). 


\section{Scheme 9: Synthesis of Compound 22}
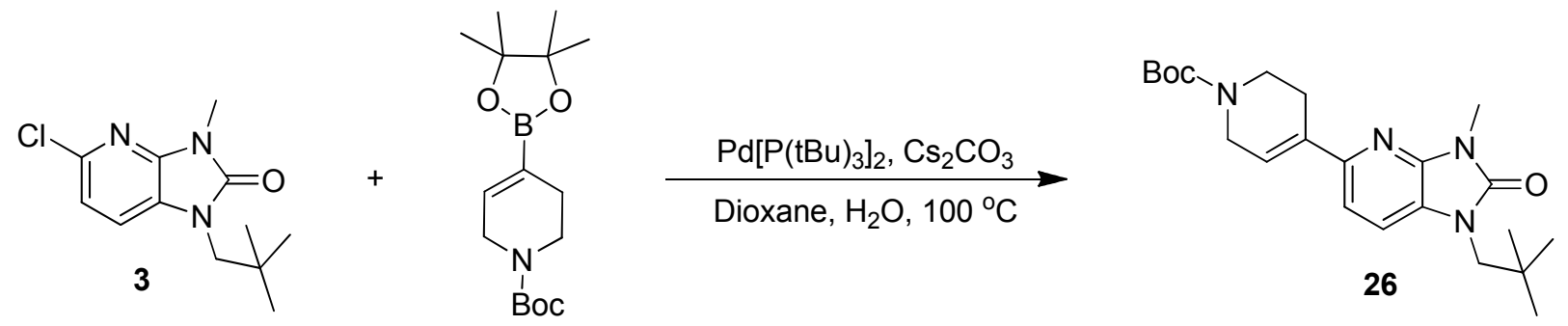

25
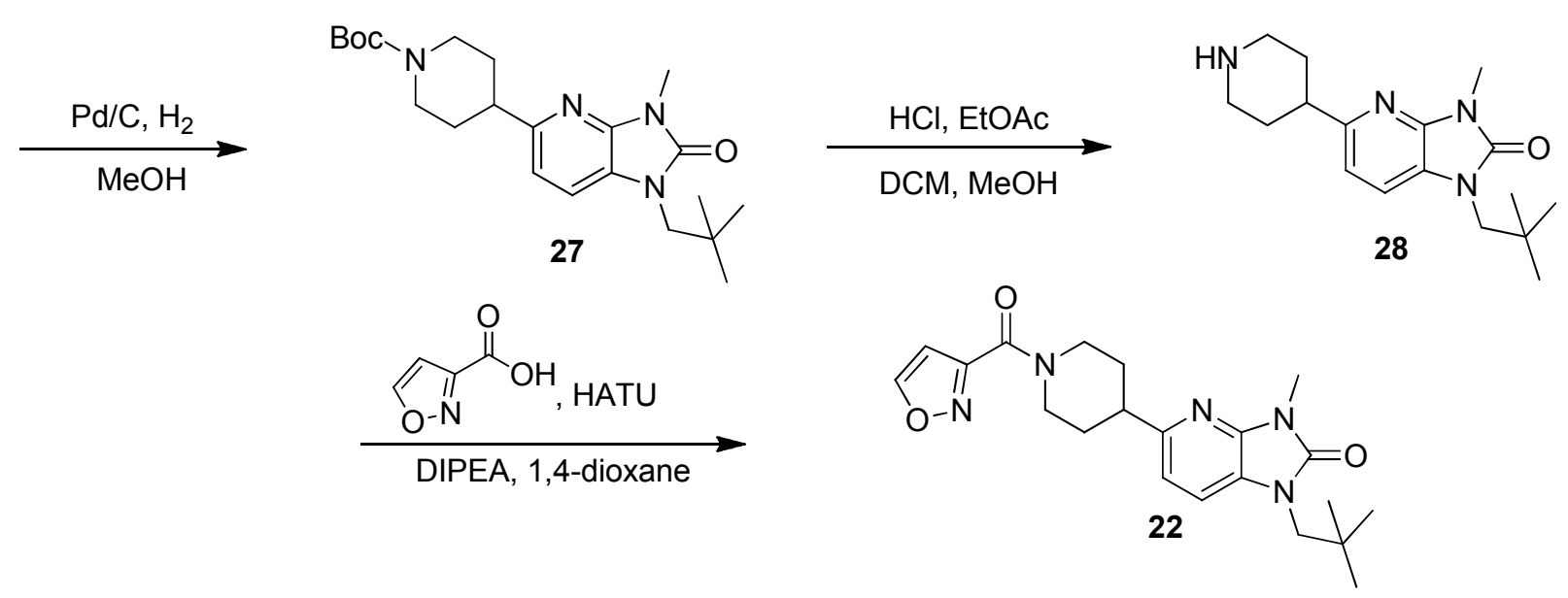

tert-Butyl-4-(3-methyl-1-neopentyl-2-oxo-2,3-dihydro- $1 H$-imidazo $[4,5$-b $]$ pyridin-5-yl)-5,6dihydropyridine-1 $(2 H)$-carboxylate (26):

1,4-Dioxane $(2.5 \mathrm{~mL})$ and water $(0.5 \mathrm{~mL})$ were added sequentially to 5-chloro-1-(2,2dimethylpropyl)-3-methyl-1,3-dihydro-2H-imidazo[4,5-b]pyridin-2-one (3, $302 \mathrm{mg}, 1.19 \mathrm{mmol})$, tert-butyl 4-(4,4,5,5-tetramethyl-1,3,2-dioxaborolan-2-yl)-3,6-dihydropyridine-1(2H)carboxylate $(\mathbf{2 5}, 395 \mathrm{mg}, 1.28 \mathrm{mmol})$, cesium carbonate $(778 \mathrm{mg}, 2.39 \mathrm{mmol})$ and bis(tri-tertbutylphosphine)palladium(0) $(82 \mathrm{mg}, 0.16 \mathrm{mmol})$. The resulting mixture was then heated to 100 ${ }^{\circ} \mathrm{C}$ and progress was followed by LC/MS. Upon complete consumption of starting material, the reaction mixture was diluted with $1 \mathrm{~mL}$ of $\mathrm{MeOH} / \mathrm{NMP} /$ water $(1: 1: 1)$ and filtered. The resulting filtrate was directly purified by reverse phase HPLC $\left(20\right.$ min gradient, $10-100 \% \mathrm{CH}_{3} \mathrm{CN}: \mathrm{H}_{2} \mathrm{O}$, $0.1 \%$ TFA as modifier) and the product-containing fractions were combined and concentrated in vacuo. The resulting residue was then re-dissolved in $5 \mathrm{~mL} \mathrm{MeOH/DCM} \mathrm{(1:1)} \mathrm{and} \mathrm{concentrated}$ in vacuo to give tert-butyl-4-(3-methyl-1-neopentyl-2-oxo-2,3-dihydro-1H-imidazo[4,5b]pyridin-5-yl)-5,6-dihydropyridine-1(2H)-carboxylate (26) as a white solid (365.1 $\mathrm{mg}, 77 \%$ ). LRMS m/z $(\mathrm{M}+\mathrm{H})$ : calculated $=401.5 ;$ observed $=401.1$. 
tert-Butyl-4-(3-methyl-1-neopentyl-2-oxo-2,3-dihydro-1 $H$-imidazo[4,5-b]pyridin-5yl)piperidine-1-carboxylate (27):

To tert-butyl-4-(3-methyl-1-neopentyl-2-oxo-2,3-dihydro-1H-imidazo[4,5-b]pyridin-5-yl)-5,6dihydropyridine-1(2H)-carboxylate $(\mathbf{2 6}, 189 \mathrm{mg}, 0.47 \mathrm{mmol})$ was added $\mathrm{MeOH}(5 \mathrm{~mL})$ followed by $10 \%$ by weight palladium on carbon $(54 \mathrm{mg}, 0.05 \mathrm{mmol})$. A balloon containing hydrogen was attached and the reaction mixture was purged $3 \mathrm{x}\left(\mathrm{H}_{2} /\right.$ vacuum) and then stirred at room temperature under an atmosphere of hydrogen. After 45 minutes, the reaction mixture was diluted with $5 \mathrm{~mL}$ of MeOH:DCM (1:1) and filtered through a pad of Celite. The filtrate was then concentrated in vacuo to afford tert-butyl-4-(3-methyl-1-neopentyl-2-oxo-2,3-dihydro-1Himidazo[4,5-b]pyridin-5-yl)piperidine-1-carboxylate (27, $181 \mathrm{mg}, 95 \%)$, which was carried forward without further purification. $\mathrm{LRMS} \mathrm{m} / \mathrm{z}(\mathrm{M}+\mathrm{H})$ : calculated $=403.5$; observed $=403.1$.

\section{3-Methyl-1-neopentyl-5-(piperidin-4-yl)-1H-imidazo[4,5-b]pyridin-2(3H)-one (28):}

To a solution of tert-butyl-4-(3-methyl-1-neopentyl-2-oxo-2,3-dihydro-1H-imidazo[4,5b]pyridin-5-yl)piperidine-1-carboxylate $(27,159 \mathrm{mg}, 0.40 \mathrm{mmol})$ in DCM (3 mL) and $\mathrm{MeOH}(3$ $\mathrm{mL})$ was added a saturated solution of $\mathrm{HCl}$ in EtOAc $(0.99 \mathrm{~mL}, 3.95 \mathrm{mmol}, \sim 4 \mathrm{~N})$. The resulting reaction mixture was stirred at room temperature. After 1 hour the reaction mixture was concentrated in vacuo to provide 3-methyl-1-neopentyl-5-(piperidin-4-yl)-1H-imidazo[4,5b]pyridin-2(3H)-one (28, $161.4 \mathrm{mg})$, which was carried forward without further purification. HRMS m/z $(\mathrm{M}+\mathrm{H})$ : calculated = 303.2181; observed = 303.2178.

5-(1-(Isoxazole-3-carbonyl)piperidin-4-yl)-3-methyl-1-neopentyl-1H-imidazo[4,5-b]pyridin$\underline{2(3 H)-o n e ~(22): ~}$

Added 3-methyl-1-neopentyl-5-(piperidin-4-yl)-1H-imidazo[4,5-b]pyridin-2(3H)-one (28, 53 $\mathrm{mg}, 0.16 \mathrm{mmol})$, isoxazole-3-carboxylic acid (64 mg, $0.57 \mathrm{mmol})$ and HATU (138 $\mathrm{mg}, 0.363$ mmol) to anhydrous 1,4-dioxane $(1 \mathrm{~mL})$ followed by DIPEA $(0.1 \mathrm{~mL}, 0.57 \mathrm{mmol})$. The resulting reaction mixture was stirred at room temperature. After 5 min, LCMS showed complete consumption of starting material. The reaction mixture was diluted with 1:1 $\mathrm{MeOH} / \mathrm{NMP}(1 \mathrm{~mL})$ and directly purified by reverse phase HPLC (20 min gradient, 10-100\% $\mathrm{CH}_{3} \mathrm{CN}: \mathrm{H}_{2} \mathrm{O}, 0.1 \%$ TFA as modifier). The product-containing fractions were combined and 
concentrated in vacuo. The resulting residue was then re-dissolved in $5 \mathrm{~mL} \mathrm{MeOH/DCM} \mathrm{(1:1)}$ and concentrated in vacuo to afford 5-(1-(isoxazole-3-carbonyl)piperidin-4-yl)-3-methyl-1neopentyl-1H-imidazo[4,5-b]pyridin-2(3H)-one (22) as a white solid (43.3 $\mathrm{mg}, 63.8 \%$ over 2 steps). $\mathrm{HRMS} \mathrm{m} / \mathrm{z}(\mathrm{M}+\mathrm{H})$ : calculated $=398.2187$; observed $=398.4789 .{ }^{1} \mathrm{H} \mathrm{NMR}(400 \mathrm{MHz}$, $\left.\mathrm{CH}_{3} \mathrm{OH}-d_{4}\right): \delta 8.78(\mathrm{~d}, 1 \mathrm{H}), 7.53(\mathrm{~d}, 1 \mathrm{H}), 7.07(\mathrm{~d}, 1 \mathrm{H}), 6.70(\mathrm{~d}, 1 \mathrm{H}), 4.27(\mathrm{~d}, 1 \mathrm{H}), 3.68(\mathrm{~s}$, $2 \mathrm{H}), 3.48(\mathrm{~s}, 3 \mathrm{H}), 3.30-3.33(\mathrm{~m}, 1 \mathrm{H}), 3.08-3.14(\mathrm{~m}, 1 \mathrm{H}), 2.98-3.04(\mathrm{~m}, 1 \mathrm{H}), 2.06(\mathrm{~d}, 1 \mathrm{H})$, 1.86-2.00 (m, $2 \mathrm{H}), 0.99$ (s, $9 \mathrm{H})$.

\section{VCD Measurements and Calculations for $(1 S, 4 S)$-eutomer of 18:}

Sample was dissolved in $\mathrm{CDCl}_{3}(50 \mathrm{mg} / \mathrm{mL}$ for each sample). All experiments were performed using a $0.10-\mathrm{mm}$ path length cell with $\mathrm{BaF}_{2}$ windows. The IR and VCD spectra were recorded using a Chiral $I R^{\mathrm{TM}}$ VCD spectrometer equipped with the Dual PEM accessory (BioTools, Jupiter, FL), with $4 \mathrm{~cm}^{-1}$ resolution. A dry $\mathrm{N}_{2}$ purge was used to eliminate water from the instrument. Data was collected in blocks, where the instrument recorded 3000 scans over the course of $1 \mathrm{~h}$ and averaged those scans into one block. Each of the runs involved averaging four blocks for each sample, as well as the solvent. The solvent background average was then subtracted from the sample average. Collection times for sample and solvent ranged were approximately five hours each. This was carried out for both pairs of enantiomers that were analyzed.

The general approach for VCD assignment at Merck, including the details computational workflow, has been published elsewhere. ${ }^{1,2}$ A subset of the details of the computational methodology is provided here. Conformers of each test structure were geometry optimized at the B3LYP/6-31G** level and stationary points were confirmed by performing frequency calculations. $^{3-11}$ All calculations were performed using Gaussian 09. ${ }^{12}$ Frequency calculations output the IR and VCD spectra. ${ }^{13}$ Frequencies were scaled by a value of 0.98 , but owing to the secondary scaling and shifting of the calculated VCD and IR spectra (during extraction of VCD by BioTools ViewVCD and during spectra alignment detailed below) in comparison to the experimental spectra, this initial scaling was, to some extent, arbitrary. 
Output conformers were ranked according to DFT energy and a clustering was performed to remove duplicates. Initial identification of duplicates was performed solely on an electronic energy basis where compounds were considered identical if the difference in Hartrees was less than 0.01. Rounding the differences led to inconsistencies in identification of duplicates. It became better to cluster the DFT minima by energy and then re-cluster each energy bucket by structure using an all atom RMS of $0.6 \AA$. This faithfully removed only identical compounds. Two Boltzmann distributions were calculated based on electronic energy (E) and free energy (G).

The in-house method for comparing VCD and IR spectra is based on published methodology. ${ }^{14}$ We used the same formulas for calculating similarity for IR and VCD spectra of experimental and observed curves. Based on our experience in matching the curves by hand we introduced the following modifications to the algorithm: we scale the spectra ( 0 to 1 for IR, -1 to 1 for VCD) before comparing them; we isolate each peak for movement rather than groups of peaks; we isolate peaks independently for IR and VCD spectra; when looking for the best match we move the experimental peak only to higher frequencies with a maximum shift of $20 \mathrm{~cm}^{-1}$; if the user sees that the baseline of the spectrum is not corrected the user can ask the program to correct the baseline. For all figures contained in the manuscript, the output intensities from Gaussian for IR $\Delta \varepsilon$ (molar absorptivity) and VCD $\Delta \varepsilon$ are D $\left(10^{-40} \mathrm{esu}^{2} \mathrm{~cm}^{2}\right)$ and $\mathrm{R}\left(10^{-44} \mathrm{esu}^{2} \mathrm{~cm}^{2}\right)$, respectively. However, owing to scaling all peaks the intensities are labeled only as 'scaled'.

Calculations of the VCD and IR spectra involved modeling the $R R$ configuration. Since the assignments indicated the actual sample to be $S S$, it should be noted that the designated absolute configuration is the enantiomeric form of the images of the conformational minima shown below.

Figure S1 provides the overlays of the calculated and measured IR and VCD spectra. A high degree of confidence is derived from the statistical and visual matching of the spectra. Assigned absolute configuration of the desired compound is provided in Scheme S1. 


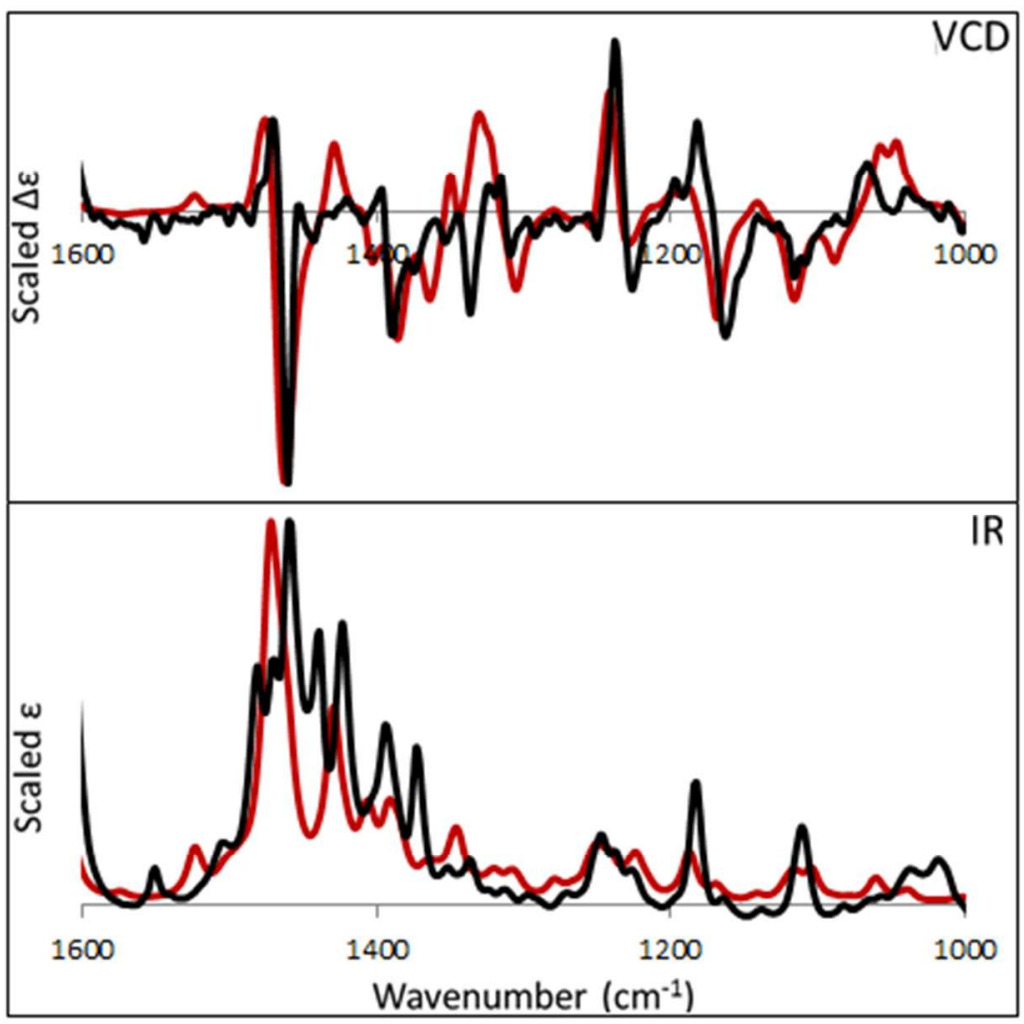

Figure S1: Overlay of measured (black) and calculated (red) VCD and IR spectra.<smiles>Cn1c(=O)n(CC(C)(C)C)c2ccc(N3CCN(C(=O)c4ccon4)CC3[Si])nc21</smiles>

$(1 S, 4 S)-18$

Scheme S1: Assigned absolute configuration SS for compound $(1 S, 4 S)-18$. 


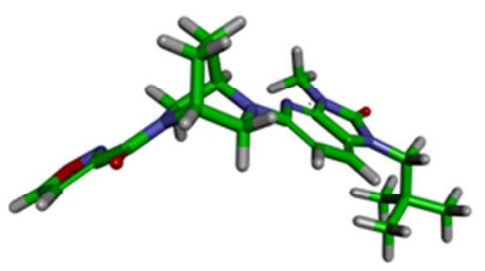

Conformer 1

$15.0 \%$

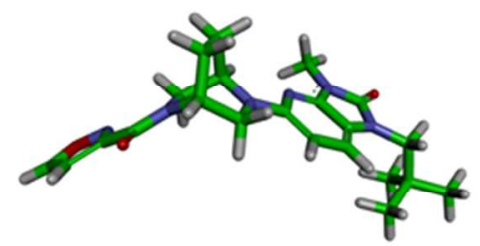

Conformer 4

$13.5 \%$

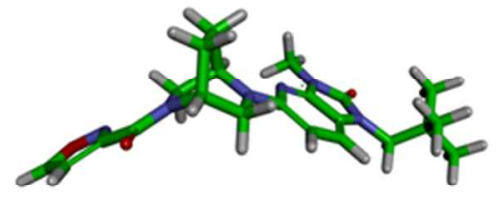

Conformer 2

$14.2 \%$

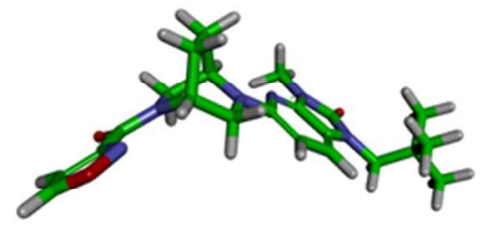

Conformer 5

$13.1 \%$

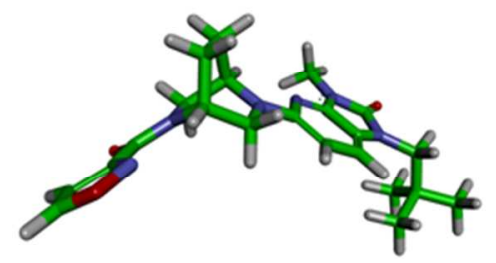

Conformer 7

$12.5 \%$

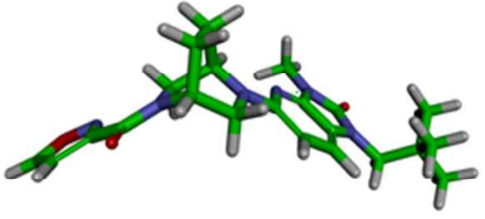

Conformer 3 $14.2 \%$

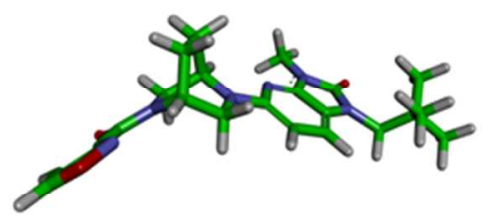

Conformer 6 $13.0 \%$

Figure S2: Conformers of the $R R$ minima contributing $>5 \%$ to the Boltzmann distribution, percentages shown above based on in vacuo electronic energies.

Coordinates and electronic energies for B3LYP/6-31G** conformational minima contributing $>5 \%$ to the in vacuo Boltzmann distribution.

Conformer 1: -1409.434415

$60.933735-0.762982-1.63841$

$60.20261-1.00327-0.451747$

$70.785174-0.9804090 .772131$

$62.07886-0.71630 .789812$

$62.89738-0.449456-0.324864$

$62.30367-0.479384-1.57554$

$65.4233-0.066374-0.606306$

$65.834931 .38706-0.961809$

$64.808132 .03701-1.90638$

$67.199771 .30049-1.6722$

65.971752 .234640 .315657

$74.1913-0.223140 .157079$

$64.20284-0.3890421 .54312$ 
$72.88019-0.662121 .91522$

$62.46996-0.8984913 .28383$

$85.1713-0.3218382 .28648$

$7-1.14809-1.28667-0.489986$

$6-1.96297-1.471990 .715309$

$6-3.02797-0.3639420 .771537$

$7-3.83296-0.479118-0.462457$

$6-3.37813-1.54419-1.37636$

$6-1.90921-1.274-1.73536$

$6-2.65124-2.848210 .644654$

$6-3.50001-2.90028-0.657997$

$6-4.894310 .292648-0.832985$

$8-5.498530 .137148-1.89699$

$6-5.375971 .388730 .074452$

$6-6.471432 .23565-0.272209$

$6-6.576193 .065240 .794119$

$8-5.636572 .763551 .71734$

$7-4.875291 .696811 .25521$

$13.817982 .09673-1.44461$

$15.116073 .05647-2.16303$

$14.714711 .47637-2.84439$

$17.142940 .687843-2.57976$

$17.544322 .29727-1.96627$

$17.962580 .863823-1.0181$

16.662871 .775981 .0283

16.340373 .236260 .068375

15.008482 .349580 .82134

$11.7203-0.1649893 .5945$

$12.04561-1.900953 .39177$

$13.35969-0.8049433 .90726$

$16.21326-0.5182170 .001116$

$15.32543-0.65792-1.52475$

$1-3.65898-0.481351 .65785$

$1-2.554650 .619990 .835039$

$1-1.82081-0.305622-2.24937$

$1-1.55203-2.05036-2.4293$

$1-3.27779-2.990871 .53196$

$1-1.8893-3.63240 .655789$

$1-4.55365-3.10046-0.443705$

$1-3.15134-3.69248-1.32928$

$1-1.30449-1.392121 .57812$

$1-4.00342-1.49427-2.2661$

$10.444585-0.793731-2.6028$

$12.86436-0.288702-2.48401$

$1-7.053342 .19401-1.17757$

$1-7.232653 .885291 .04476$ 
Conformer 2: -1409.434365

$60.937426-1.44405-0.661403$

$60.220221-0.5985570 .216827$

70.7751270 .5100130 .765597

62.030530 .7499750 .434131

$62.83981-0.03-0.414512$

$62.27017-1.15757-0.981919$

$65.21650 .267192-1.34719$

$66.28001-0.701811-0.765323$

$67.40468-0.807203-1.81371$

$65.67854-2.09708-0.520146$

$66.85288-0.1460770 .550809$

$74.09140 .592456-0.47922$

64.061491 .76760 .273863

72.787991 .827540 .854474

62.354382 .907241 .7167

84.959332 .588340 .397248

$7-1.08892-0.8704920 .559193$

$6-1.88692-0.0058551 .43347$

$6-3.127190 .4706920 .659421$

$7-3.87058-0.7397130 .25159$

$6-3.21758-2.002920 .644271$

$6-1.80787-2.026450 .034138$

$6-2.32598-0.8126272 .67036$

$6-3.12341-2.055892 .18008$

$6-5.04995-0.796547-0.430348$

$8-5.5986-1.85834-0.735188$

$6-5.738920 .47353-0.841731$

$6-6.972160 .471157-1.56052$

$6-7.240841 .78894-1.7267$

$8-6.268392 .54053-1.16481$

$7-5.315231 .69971-0.603251$

$17.872940 .166549-1.99485$

$18.18577-1.49424-1.47225$

$17.02737-1.18323-2.77214$

$15.29479-2.53663-1.44884$

$16.43975-2.77844-0.124752$

$14.85923-2.060 .204135$

$16.08643-0.1012821 .33013$

$17.661-0.7905950 .914036$

17.247510 .8654080 .420391

11.496153 .427451 .28149

12.072 .52442 .70114

13.193083 .596961 .81602

$14.81159-0.146804-2.27868$

$15.698331 .22032-1.58469$ 
$1-3.749781 .113041 .28963$

$1-2.831461 .05665-0.215281$

$1-1.87253-1.99518-1.06348$

$1-1.30571-2.966810 .308895$

$1-2.93851-0.1808623 .32298$

$1-1.44007-1.106253 .2401$

$1-4.13239-2.078162 .60156$

$1-2.63021-2.988052 .4763$

$1-1.273080 .85051 .70596$

$1-3.8203-2.815660 .242709$

$10.466337-2.31736-1.09219$

$12.82275-1.80716-1.65182$

$1-7.5283-0.394742-1.87827$

$1-8.04282 .33727-2.19834$

Conformer 3: -1409.434365

$60.917699-1.46835-0.705864$

$60.20182-0.7359650 .269428$

70.7412170 .3291750 .911417

61.979850 .6387350 .574652

$62.78552-0.027128-0.369419$

$62.23198-1.11028-1.03102$

$65.121750 .439603-1.33417$

$66.22795-0.553908-0.889821$

$67.32035-0.515154-1.97601$

$65.67169-1.98464-0.77602$

$66.82695-0.1222550 .460688$

$74.016120 .637588-0.405096$

63.974911 .730660 .462792

72.718241 .69261 .08036

62.27952 .668592 .05622

84.8522 .563180 .642998

7 -1.08688 -1.08669 0.623608

$6-1.90524-0.2897791 .54427$

$6-3.06910 .3433120 .76239$

$7-3.84121-0.7682910 .168303$

$6-3.27038-2.102650 .434678$

$6-1.82798-2.1203-0.093679$

$6-2.46229-1.216622 .64062$

$6-3.27541-2.350981 .95337$

$6-4.97615-0.68015-0.582178$

$8-5.5524-1.66704-1.0463$

$6-5.57790 .664869-0.874748$

$6-6.757190 .814073-1.66497$

$6-6.95512 .15451-1.68306$

$8-5.991722 .78064-0.971673$ 
$7-5.117791 .82928-0.459606$ $17.758820 .485018-2.06466$ $18.12905-1.21288-1.73495$ $16.92129-0.79622-2.95787$ $15.27088-2.33599-1.73454$ $16.46276-2.6807-0.476424$ $14.87458-2.04832-0.029176$ $16.08277-0.1773821 .26074$ $17.66022-0.7790810 .733926$ 17.19390 .9073490 .424882 13.097553 .376192 .19462 11.389493 .195741 .70054 12.04332 .184123 .0081 $14.699880 .116583-2.2937$ $15.571481 .42619-1.47992$ $1-3.700230 .940441 .42768$ $1-2.68951 .0118-0.015667$ $1-1.82628-1.93448-1.17779$ $1-1.38672-3.114850 .072021$ $1-3.09221-0.636323 .32361$ $1-1.6323-1.620153 .22697$ $1-4.30875-2.378252 .31053$ $1-2.83781-3.334392 .15651$ $1-1.274050 .4944261 .95761$ $1-3.8811-2.82729-0.101052$ $10.463671-2.31497-1.20334$ $12.78403-1.67378-1.77521$ $1-7.327570 .023173-2.12233$ $1-7.697352 .79704-2.13303$

Conformer 4: -1409.434314 $6-0.9542110 .260899-1.70968$ $6-0.2256730 .730312-0.591854$ $7-0.8199790 .9974960 .596999$ $6-2.122760 .7882250 .649407$ $6-2.940780 .313993-0.394345$ $6-2.334580 .047073-1.61054$ $6-5.47722-0.026247-0.63128$ $6-5.94529-1.50552-0.666753$ $6-7.30015-1.52413-1.40108$ $6-4.93867-2.38374-1.43111$ $6-6.12514-2.047080 .762479$ $7-4.247250 .2463620 .102088$ $6-4.265270 .7133211 .41764$ $7-2.936251 .011951 .74476$ $6-2.529071 .526473 .03575$ 
$8-5.242670 .8472692 .13999$

$71.136810 .938305-0.664085$

61.934631 .428430 .463889

63.09360 .4472150 .705585

$73.87470 .385337-0.547241$

$63.328431 .22037-1.6331$

$61.884790 .771936-1.90594$

62.498462 .82070 .119636

$63.341582 .69251-1.18231$

$64.99124-0.358307-0.79111$

$85.57086-0.361881-1.87988$

$65.56823-1.221120 .294866$

$66.72098-2.036510 .085851$

$66.90475-2.633911 .28821$

$85.95768-2.228872 .16298$

$75.10971-1.330391 .52679$

$1-7.21275-1.12119-2.41718$

$1-7.68042-2.54766-1.48225$

$1-8.05076-0.930956-0.867179$

$1-5.28392-3.42287-1.46327$

$1-4.81834-2.04576-2.46759$

$1-3.95422-2.37648-0.953616$

$1-6.80275-1.41811 .34643$

$1-6.53155-3.064140 .734262$

$1-5.17055-2.083291 .29553$

$1-3.428611 .614853 .64576$

$1-1.820210 .8462833 .51693$

$1-2.056242 .506962 .92794$

$1-6.253430 .578725-0.153106$

$1-5.345880 .343705-1.65551$

13.719430 .7900221 .53534

$12.70946-0.5416050 .970884$

$11.87261-0.274009-2.24566$

$11.457851 .3869-2.71317$

13.108073 .187440 .952859

$11.669243 .522-0.008541$

$14.376053 .01045-1.0242$

$12.932443 .31535-1.98516$

11.28991 .459421 .34018

$13.95131 .05583-2.51062$

$1-0.45320 .051205-2.64526$

$1-2.89325-0.319924-2.46456$

$17.28519-2.12835-0.826986$

$17.62616-3.339631 .67261$

Conformer 5: -1409.434289

$60.77136-1.259970 .259615$ 
60.2330680 .0399720 .404827

70.9788321 .155640 .212901

$62.241910 .954794-0.115847$

$62.88087-0.289992-0.275792$

$62.11794-1.42966-0.084923$

$65.22348-0.980488-1.07052$

$66.1591-1.554220 .026337$

$67.18826-2.4484-0.692186$

$66.88973-0.4158610 .76119$

$65.36723-2.399881 .03935$

$74.21019-0.030971-0.627152$

$64.397771 .34819-0.735911$

$73.17711 .93785-0.382158$

$62.9573 .36919-0.371728$

$85.416631 .9296-1.08049$

$7-1.087410 .2296230 .761816$

$6-1.713321 .555250 .843396$

$6-2.795171 .6588-0.24095$

$7-3.767030 .5747210 .003515$

$6-3.41862-0.2966821 .14109$

$6-2.02199-0.8847010 .877907$

$6-2.361771 .710762 .23229$

$6-3.387940 .5574882 .42336$

$6-4.83250 .512833-0.839979$

$8-4.971291 .32783-1.75444$

$6-5.89562-0.546004-0.703915$

$6-6.99313-0.622227-1.61342$

$6-7.71003-1.6774-1.15593$

$8-7.11803-2.20943-0.064445$

$7-5.96576-1.485970 .21824$

$17.78433-1.87312-1.40925$

$17.87854-2.898040 .029044$

$16.70089-3.2649-1.23815$

$17.6092-0.8270381 .47786$

17.427130 .2315420 .062616

16.188480 .213241 .31734

$14.86322-3.242720 .5511$

$16.03825-2.814731 .79945$

$14.60983-1.802831 .55621$

$12.17443 .64479-1.08466$

12.6583 .706350 .624954

$13.897823 .84173-0.655621$

$14.7088-1.80302-1.5819$

$15.83015-0.451745-1.81166$

$1-3.305632 .62804-0.205636$

$1-2.364751 .55403-1.24233$

$1-2.03942-1.49004-0.040499$

$1-1.74706-1.55251 .70847$ 
$1-2.849722 .68922 .29989$

$1-1.58341 .681942 .99974$

$1-4.392740 .9412052 .6222$

$1-3.11511-0.0829443 .26893$

$1-0.939052 .300220 .671054$

$1-4.14777-1.098781 .21785$

$10.153401-2.133390 .419541$

$12.5333-2.42562-0.192767$

$1-7.174250 .026937-2.4535$

$1-8.62252-2.15888-1.47493$

Conformer 6: -1409.434277

$60.803945-1.25631-0.144166$

60.2585510 .0234340 .112014

71.018621 .146020 .107778

$62.302730 .97119-0.145398$

$62.95143-0.253629-0.395831$

$62.17318-1.39925-0.40075$

$65.36018-0.867922-1.05739$

$66.17863-1.583590 .050347$

$67.30509-2.35823-0.661125$

$65.29845-2.57130 .83687$

$66.7916-0.5538681 .01619$

$74.30530 .031129-0.605657$

$64.502641 .41145-0.534574$

$73.256961 .96895-0.219535$

$63.03773 .3913-0.058275$

$85.548162 .01793-0.71971$

$7-1.086330 .1874280 .374378$

$6-1.698711 .493190 .644085$

$6-2.892651 .66939-0.303335$

$7-3.833150 .562827-0.037467$

$6-3.35546-0.3956760 .975704$

$6-1.99591-0.9443950 .510159$

6 -2.18969 1.530452 .10507

$6-3.181070 .3498242 .31395$

$6-5.001340 .575534-0.734138$

$8-5.245871 .46115-1.55609$

$6-6.05435-0.484074-0.539425$

$6-7.24378-0.508935-1.32796$

$6-7.92305-1.57606-0.841548$

$8-7.22593-2.161210 .156696$

$7-6.03966-1.462040 .345015$

$17.97013-1.6801-1.2072$

$17.91302-2.907680 .065039$

$16.90449-3.08612-1.37658$

$14.87537-3.341060 .18003$ 
$15.88885-3.083431 .60446$ $14.47209-2.061111 .34082$ $16.01486-0.0219091 .57379$ $17.43974-1.055071 .74351$ 17.382660 .1946320 .481328 $13.997463 .88486-0.214081$ $12.31093 .75485-0.790513$ 12.66493 .613320 .945887 $14.90587-1.61323-1.72158$ $16.03943-0.254166-1.65637$ $1-3.395992 .62836-0.136563$ $1-2.574581 .64485-1.35079$

$1-2.11398-1.48433-0.441024$ $1-1.62436-1.664541 .25497$ $1-2.672222 .493182 .30641$ $1-1.329551 .452242 .77578$ $1-4.158380 .7005572 .65753$ $1-2.80958-0.3547193 .06584$ $1-0.9498072 .258870 .451101$ 1 -4.07017-1.20907 1.06808 $10.170684-2.13341-0.152329$ $12.59315-2.3801-0.594603$ $1-7.505140 .179628-2.11379$ $1-8.8715-2.03368-1.0808$

Conformer 7: -1409.434247 $6-0.789499-0.80449-1.20528$ $6-0.2445920 .388366-0.675053$ $7-1.01721 .33882-0.09392$ $6-2.311491 .08086-0.048732$ $6-2.95567-0.074743-0.531618$ $6-2.16747-1.04211-1.13199$ $6-5.42306-0.768991-0.722161$ $6-5.86146-1.902010 .243587$ $6-7.09171-2.57735-0.393396$ $6-6.24459-1.320621 .61617$ $6-4.7415-2.943530 .417182$ $7-4.322610 .075013-0.273068$ $6-4.546251 .31870 .320188$ $7-3.285661 .909430 .476873$ $6-3.085923 .217371 .06551$ $8-5.624021 .804470 .63246$ $71.110750 .64282-0.73711$ $61.732451 .83891-0.157568$ 62.706171 .402270 .946021 73.698620 .5021850 .325392 $63.469080 .257468-1.11057$ 
$62.0567-0.329231-1.27256$

$62.509232 .58024-1.26282$

$63.561421 .60191-1.85925$

64.684110 .0316561 .13634

84.740590 .3522872 .32536

$65.75479-0.9001450 .630487$

$66.80105-1.365471 .48263$

$67.53559-2.166370 .672915$

$86.99962-2.19924-0.566648$

$75.86805-1.39286-0.587474$

$1-6.85859-2.98659-1.38363$

$1-7.44388-3.404010 .232192$

$1-7.9203-1.86976-0.508367$

$1-7.01033-0.5453511 .52372$

$1-6.62845-2.112022 .26944$

$1-5.37969-0.8695812 .11153$

$1-3.84099-2.497980 .85023$

$1-5.06753-3.748221 .08507$

$1-4.46934-3.40218-0.54116$

$1-2.438743 .149711 .94471$

$1-2.62723 .89990 .344057$

$1-4.067523 .59281 .35624$

$1-6.27034-0.096512-0.885875$

1 -5.1422 -1.19864 -1.69149

13.215762 .263341 .39326

12.183010 .8782251 .75265

$11.98812-1.28967-0.74092$

$11.86688-0.526961-2.33864$

$12.990463 .4685-0.839129$

$11.807672 .92438-2.02776$

$14.57731 .99609-1.76382$

$13.38381 .4269-2.92577$

10.9403052 .454660 .263796

$14.20568-0.452558-1.47688$

$1-0.150694-1.54391-1.66947$

1 -2.58597 -1.95856-1.53317

$16.9386-1.116242 .52133$

$18.42671-2.759990 .813352$

\section{References}

1. E.C. Sherer, C.H. Lee, J. Shpungin, J.F. Cuff, C.X. Da, R. Ball, R. Bach, A. Crespo, X.Y. Gong, and C.J. Welch, Journal of Medicinal Chemistry, 2014, 57(2), 477. 
2. Z.Q. Liu, C.S. Shultz, C.A. Sherwood, S. Krska, P.G. Dormer, R. Desmond, C. Lee, E.C. Sherer, J. Shpungin, J. Cuff, and F. Xu, Tetrahedron Letters, 2011, 52(14), 1685.

3. G.A. Petersson, and M.A. Allaham, Journal of Chemical Physics, 1991, 94(9), 6081.

4. G.A. Petersson, A. Bennett, T.G. Tensfeldt, M.A. Allaham, W.A. Shirley, and J. Mantzaris, Journal of Chemical Physics, 1988, 89(4), 2193.

5. V.A. Rassolov, J.A. Pople, M.A. Ratner, and T.L. Windus, Journal of Chemical Physics, 1998, 109(4), 1223.

6. V.A. Rassolov, M.A. Ratner, J.A. Pople, P.C. Redfern, and L.A. Curtiss, Journal of Computational Chemistry, 2001, 22(9), 976.

7. M.M. Francl, W.J. Pietro, W.J. Hehre, J.S. Binkley, M.S. Gordon, D.J. Defrees, and J.A. Pople, Journal of Chemical Physics, 1982, 77(7), 3654.

8. W.J. Hehre, R. Ditchfield, and J.A. Pople, J. Chem. Phys, 1972, 56, 2257.

9. A.D. Becke, J. Chem. Phys., 1993, 98, 5648.

10. C.T. Lee, W.T. Yang, and R.G. Parr, Physical Review B, 1988, 37(2), 785.

11. B. Miehlich, A. Savin, H. Stoll, and H. Preuss, Chemical Physics Letters, 1989, 157(3), 200.

12. M.J. Frisch, G.W. Trucks, H.B. Schlegel, G.E. Scuseria, M.A. Robb, J.R. Cheeseman, G. Scalmani, V. Barone, B. Mennucci, G.A. Petersson, H. Nakatsuji, M. Caricato, X. Li, H.P. Hratchian, A.F. Izmaylov, J. Bloino, G. Zheng, J.L. Sonnenberg, M. Hada, M. Ehara, K. Toyota, R. Fukuda, J. Hasegawa, M. Ishida, T. Nakajima, Y. Honda, O. Kitao, H. Nakai, T. Vreven, J. Montgomery, J.A., J.E. Peralta, F. Ogliaro, M. Bearpark, J.J. Heyd, E. Brothers, K.N. Kudin, V.N. Staroverov, R. Kobayashi, J. Normand, K. Raghavachari, A. Rendell, J.C. Burant, S.S. Iyengar, J. Tomasi, M. Cossi, N. Rega, J.M. Millam, M. Klene, J.E. Knox, J.B. Cross, V. Bakken, C. Adamo, J. Jaramillo, R. Gomperts, R.E. Stratmann, O. Yazyev, A.J. Austin, R. Cammi, C. Pomelli, J.W. Ochterski, R.L. Martin, K. Morokuma, V.G. Zakrzewski, G.A. Voth, P. Salvador, J.J. Dannenberg, S. Dapprich, A.D. Daniels, Ö. Farkas, J.B. Foresman, J.V. Ortiz, J. Cioslowski, and D.J. Fox. Gaussian 09, Revision A.02. Wallingford, CT: Gaussian, Inc.; 2009.

13. J.R. Cheeseman, M.J. Frisch, F.J. Devlin, and P.J. Stephens, Chemical Physics Letters, 1996, 252(34), 211.

14. J.A. Shen, C.Y. Zhu, S. Reiling, and R. Vaz, Spectrochimica Acta Part a-Molecular and Biomolecular Spectroscopy, 2010, 76(3-4), 418. 